\title{
Status and prospects of the nMSSM after LHC Run-1
}

\author{
D. Barducci, ${ }^{a}$ G. Bélanger, ${ }^{a}$ C. Hugonie ${ }^{b}$ and A. Pukhov ${ }^{c}$ \\ ${ }^{a}$ LAPTh, Université de Savoie Mont Blanc, CNRS, \\ B.P. 110, F-74941 Annecy-le-Vieux, France \\ ${ }^{b}$ LUPM, UMR 5299, CNRS, Université de Montpellier, \\ 34095 Montpellier, France \\ ${ }^{c}$ Skobeltsyn Institute of Nuclear Physics, Moscow State University, \\ Moscow 119992, Russia \\ E-mail: barducci@lapth.cnrs.fr, belanger@lapth.cnrs.fr, \\ cyril.hugonie@umontpellier.fr, pukhov@lapth.cnrs.fr
}

ABStract: The new minimal supersymmetric standard model (nMSSM), a variant of the general next to minimal supersymmetric standard model (NMSSM) without $Z_{3}$ symmetry, features a naturally light singlino with a mass below $75 \mathrm{GeV}$. In light of the new constraints from LHC Run-1 on the Higgs couplings, sparticles searches and flavour observables, we define the parameter space of the model which is compatible with both collider and dark matter (DM) properties. Among the regions compatible with these constraints, implemented through NMSSMTools, SModelS and MadAnalysis5, only one with a singlino lightest supersymmetric particle (LSP) with a mass around $5 \mathrm{GeV}$ can explain all the DM abundance of the universe, while heavier mixed singlinos can only form one of the DM components. Typical collider signatures for each region of the parameter space are investigated. In particular, the decay of the $125 \mathrm{GeV}$ Higgs into light scalars and/or pseudoscalars and the decay of the heavy Higgs into charginos and neutralinos, provide distinctive signatures of the model. Moreover, the sfermion decays usually proceed through heavier neutralinos rather than directly into the LSP, as the couplings to the singlino are suppressed. We also show that direct detection searches are complementary to collider ones, and that a future ton-scale detector could completely probe the region of parameter space with a LSP mass around $65 \mathrm{GeV}$.

KeYwords: Supersymmetry Phenomenology

ARXIV EPRINT: 1510.00246 


\section{Contents}

1 Introduction 1

2 The nMSSM 3

$\begin{array}{llr}3 & \text { Parameter scan } & 7\end{array}$

4 LHC and DM phenomenology $\quad 10$

4.1 Region $1, m_{\tilde{\chi}_{1}^{0}}<5 \mathrm{GeV}$

$\begin{array}{lll}\text { 4.1.1 Current constraints } & 11\end{array}$

4.1.2 LHC Run-2 and direct detection experiments prospects 14

$\begin{array}{lll}4.2 & \text { Region 2, } 45<m_{\tilde{\chi}_{1}^{0}}<55 \mathrm{GeV} & 17\end{array}$

4.3 Region $3, m_{\tilde{\chi}_{1}^{0}} \sim 65 \mathrm{GeV} 20$

4.3.1 Region 3A and 3B: bino or higgsino/singlino LSP with low $M_{1 / 2} \quad 21$

4.3.2 Region 3C: higgsino/singlino LSP with high $M_{1 / 2} \quad 22$

5 Conclusions $\quad 24$

\section{Introduction}

The discovery of a Higgs boson with a mass of $125 \mathrm{GeV}$ at the Large Hadron Collider (LHC) $[1,2]$ can be viewed as an argument in favour of supersymmetry (SUSY) since a light Higgs boson is a landmark of this theory. However the mass of the new particle is only within a few $\mathrm{GeV}$ of the maximum value predicted in the minimal supersymmetric standard model (MSSM) and requires large contributions from the stop sector, thus raising the issue of fine-tuning [3, 4]. In the next-to-minimal supersymmetric extension of the standard model, the NMSSM, ${ }^{1}$ the fine-tuning issue is not as severe because of additional contributions to the lightest Higgs doublet mass, derived from the extra singlet superfield [7-15]. The NMSSM has the nice additional feature that the $\mu$ term is generated from the vacuum expectation value (VEV) of the new singlet field and is thus naturally at the SUSY scale, therefore solving the so-called $\mu$-problem [16]. For these reasons the discovery of the Higgs at the LHC has triggered a renewed interest in the NMSSM and phenomenological studies abound [17-25]. The main focus has been on the Higgs sector, since the extra singlet can lead to new collider signatures, in particular when light, as the Standard Model (SM) like Higgs state $h$ with $m_{h} \sim 125 \mathrm{GeV}$ can decay into light singlet like scalars or pseudoscalars. Moreover its spin $1 / 2$ SUSY partner, the singlino, can be at a mass well below the electroweak (EW) scale, giving also rise to peculiar SUSY signatures, especially when it is the lightest SUSY particle (LSP) and very light $[19,22,25,26]$. The

\footnotetext{
${ }^{1}$ For a review, see refs. $[5,6]$.
} 
NMSSM also provides a dark matter (DM) candidate, the LSP neutralino, and its properties have been analysed, both in the NMSSM with arbitrary parameters at the SUSY scale $[27,28]$ and in the grand unified theory (GUT) scale constrained models [29-31]. In general the predictions are similar to those of the MSSM, but special features including the possibility of a light neutralino LSP with an important singlino component, can increase the annihilation into Higgs final states or resonant annihilation through a singlet Higgs. Light neutralinos LSP can also escape astrophysical constraints [32-34].

These studies were conducted within the framework of the $Z_{3}$ invariant NMSSM. However the superpotential of the general NMSSM does not necessarily possess this accidental $Z_{3}$ symmetry. The new minimal supersymmetric model (nMSSM), sometimes also called minimal next-to-minimal supersymmetric model (MNSSM) [35-37], features instead a global discrete R-symmetry which forbids the singlet cubic self interaction in the superpotential, while avoiding problems with domain walls due to the $Z_{3}$ symmetry. Although the field content is the same as that of the ( $Z_{3}$ invariant) NMSSM, the different superpotential and soft SUSY breaking terms lead to a peculiar phenomenology [38-53]. The most striking feature of the model is that there is no mass term for the pure singlino. Only mixing effects with higgsinos can raise the singlino mass to the EW scale (up to $\sim 75 \mathrm{GeV}$ [54]). The singlino is therefore naturally light, and the LSP generally contains a large singlino component, thus guaranteeing a phenomenology rather different from that of the MSSM both at colliders and in DM searches. Yet, all the phenomenological studies of the nMSSM have overlooked the possibility of a very light singlino LSP (below $5 \mathrm{GeV}$ ) in agreement with DM constraints. In addition, the results from LHC Run-1 and the prospects for Run-2 are still unexplored in this model. This is the gap we intend to fill here.

In this paper we explore the parameter space of the nMSSM with unified conditions at the GUT scale, that is compatible with the latest Higgs results, with LHC searches for SUSY particles in Run-1 and with DM constraints. For this we rely on NMSSMTools $[55,56]$ for the calculation of the spectrum and constraints on the Higgs sector, on SMode1S [57, 58] for comparisons with limits on simplified models, on MadAnalysis5 [59-61] for a more complete implementation of LHC searches for sparticles and on micrOMEGAs [62-64] for the computation of the DM observables including relic density, direct detection (DD) and indirect detection (ID).

We show that this combined set of requirements lead to strong constraints on the model. We found that the allowed regions have very specific characteristics: they contain either a very light singlino LSP below $5 \mathrm{GeV}$, a mixed singlino-higgsino LSP with a mass around $45 \mathrm{GeV}$ or $65 \mathrm{GeV}$, or a bino LSP with a mass around $65 \mathrm{GeV}$. We have also checked that the allowed regions in parameter space of the general model with arbitrary parameters at the SUSY scale (i.e. without unified conditions at the GUT scale) share the same general characteristics. After having discussed the main constraints on the model, we analyse for each region some distinctive signatures that can arise at the LHC Run-2. Here we consider both searches for new Higgs states and sparticles. The complementarity between collider and DD searches is also highlighted. Moreover, we investigate the potential of ID to probe this model, which turns out to be quite limited, except for very peculiar kinematics which could lead to an enhanced gamma-ray line [65-67]. 
The paper is organized as follows. Section 2 describes the model. Section 3 contains the results of the parameter scan and the description of the different allowed regions. The LHC phenomenology of each region and the complementarity with DM searches is explored in detail in section 4. Finally benchmark points corresponding to the channels with interesting signatures at the LHC are provided. Section 5 contains our conclusions.

\section{The nMSSM}

The MSSM is defined by promoting each SM field $\Phi$ into a superfield $\widehat{\Phi}$, doubling the Higgs fields with two $\mathrm{SU}(2)_{L}$ doublets $H_{u}, H_{d}$ and imposing R-parity conservation. SUSY breaking is assumed to occur in an invisible sector and to be mediated through gravitational interactions to the visible sector. The resulting theory contains a number of soft SUSY breaking terms proportional to powers of the SUSY breaking scale $M_{\text {susy }}$. Unfortunately, a realistic realisation of EW symmetry breaking in the MSSM requires the presence of the so called $\mu$-term in the superpotential, coupling directly the two Higgs fields $H_{u}$ and $H_{d}$ :

$$
W_{\mathrm{MSSM}}=\mu \widehat{H}_{u} \widehat{H}_{d}+h_{u} \widehat{Q} \widehat{U}^{c} \widehat{H}_{u}+h_{d} \widehat{Q} \widehat{D}^{c} \widehat{H}_{d}+h_{e} \widehat{L} \widehat{E}^{c} \widehat{H}_{d}
$$

with values of the arbitrary $\mu$ parameter close to $M_{\text {susy }}$. There exist explanations for such a value of the $\mu$-term, alas, all in extended settings [68]. The easiest solution to the $\mu$ problem is to introduce an extra gauge singlet $\mathrm{S}$, coupled to the Higgs doublets and whose VEV is naturally of the order of $M_{\text {susy }}$. This leads to the simplest extension of the MSSM, the NMSSM with a cubic (renormalisable) superpotential

$$
W_{\mathrm{NMSSM}}=\lambda \widehat{S} \widehat{H}_{u} \widehat{H}_{d}+\frac{1}{3} \kappa \widehat{S}^{3}+h_{u} \widehat{Q} \widehat{U}^{c} \widehat{H}_{u}+h_{d} \widehat{Q} \widehat{D}^{c} \widehat{H}_{d}+h_{e} \widehat{L} \widehat{E}^{c} \widehat{H}_{d}
$$

The presence of the singlet cubic self interaction is necessary in order to avoid a global U(1) Peccei-Quinn (PQ) symmetry which would lead to massless singlet states, experimentally excluded [6]. The corresponding soft SUSY breaking potential is given by

$$
\begin{aligned}
V_{\mathrm{NMSSM}}= & m_{H_{u}}^{2}\left|H_{u}\right|^{2}+m_{H_{d}}^{2}\left|H_{d}\right|^{2}+m_{S}^{2}|S|^{2}+\left(\lambda A_{\lambda} H_{u} H_{d} S+\frac{1}{3} \kappa A_{\kappa} S^{3}+\text { h.c. }\right) \\
& +m_{Q}^{2}\left|Q^{2}\right|+m_{U}^{2}\left|U_{R}^{2}\right|+m_{D}^{2}\left|D_{R}^{2}\right|+m_{L}^{2}\left|L^{2}\right|+m_{E}^{2}\left|E_{R}^{2}\right| \\
& +\left(h_{u} A_{u} Q H_{u} U_{R}^{c}-h_{d} A_{d} Q H_{d} D_{R}^{c}-h_{e} A_{e} L H_{d} E_{R}^{c}+\text { h.c. }\right) \\
& +M_{1} \widetilde{B} \widetilde{B}+M_{2} \widetilde{W} \widetilde{W}+M_{3} \widetilde{g} \widetilde{g} .
\end{aligned}
$$

The standard NMMSM given by eqs. (2.2)-(2.3) possesses a global $Z_{3}$ symmetry under which all superfields are multiplied by $e^{2 i \pi / 3}$. Once this discrete symmetry is spontaneously broken during the phase transition associated with the EW symmetry breaking in the early universe, cosmologically dangerous domain walls are generated [69]. It has been argued in $[70,71]$ that these walls become harmless if they disappear before nucleosynthesis, which requires the presence in the effective potential of $Z_{3}$ breaking terms of magnitude

$$
\delta V \sim O(1 \mathrm{MeV})^{4} .
$$


The magnitude of the $Z_{3}$ breaking terms corresponds to the presence in the superpotential or in the Kähler potential of $Z_{3}$ breaking operators suppressed by one inverse power of the Planck mass, $M_{\text {Planck }}$. However, these $Z_{3}$ breaking (non-renormalisable) terms involving the singlet $S$ induce divergent tadpoles [72-78] of the form

$$
\delta W=\Xi_{F} M_{\text {susy }} M_{\text {Planck }} S, \quad \delta V=\Xi_{S} M_{\text {susy }}^{2} M_{\text {Planck }}\left(S+S^{*}\right),
$$

thus reintroducing a hierarchy problem. The values of $\Xi_{F}$ and $\Xi_{S}$ depend on the loop order at which the tadpoles are generated, which in turn depends on the particular nonrenormalisable terms that give rise to the tadpoles. A solution to both the domain wall and the stability problem is to impose a discrete $R$-symmetry on the complete theory (including non-renormalisable operators) such that the tadpole terms are generated at high loop order [79]. One then obtains effective tadpole terms

$$
\delta W=\xi_{F} S, \quad \delta V=\xi_{S}\left(S+S^{*}\right), \quad \text { where } \quad \xi_{F} \lesssim M_{\text {susy }}^{2} \quad \text { and } \quad \xi_{S} \lesssim M_{\text {susy }}^{3} .
$$

In the case where $\xi_{F} \sim M_{\text {susy }}^{2}$ and $\xi_{S} \sim M_{\text {susy }}^{3}$ the singlet cubic self interaction in the superpotential (2.2) is not even phenomenologically required and can be omitted [35]. The resulting model has been denoted as the new MSSM or nMSSM as, in the limit where SUSY is unbroken, the MSSM $\mu$ term is only traded for the dimensionless $\lambda$ coupling. Once SUSY is softly broken, the generated tadpole terms $\xi_{F}$ and $\xi_{S}$ break both the $Z_{3}$ and the PQ symmetry. The superpotential of the nMSSM then reads

$$
W_{\mathrm{nMSSM}}=\lambda \widehat{S} \widehat{H}_{u} \widehat{H}_{d}+\xi_{F} \widehat{S}+h_{u} \widehat{Q} \widehat{U}^{c} \widehat{H}_{u}+h_{d} \widehat{Q} \widehat{D}^{c} \widehat{H}_{d}+h_{e} \widehat{L} \widehat{E}^{c} \widehat{H}_{d}
$$

and the corresponding soft SUSY breaking potential is given by

$$
\begin{aligned}
V_{\mathrm{nMSSM}}= & m_{H_{u}}^{2}\left|H_{u}\right|^{2}+m_{H_{d}}^{2}\left|H_{d}\right|^{2}+m_{S}^{2}|S|^{2}+\left(\lambda A_{\lambda} H_{u} H_{d} S+\xi_{S} S+\text { h.c. }\right) \\
& +m_{Q}^{2}\left|Q^{2}\right|+m_{U}^{2}\left|U_{R}^{2}\right|+m_{D}^{2}\left|D_{R}^{2}\right|+m_{L}^{2}\left|L^{2}\right|+m_{E}^{2}\left|E_{R}^{2}\right| \\
& +\left(h_{u} A_{u} Q H_{u} U_{R}^{c}-h_{d} A_{d} Q H_{d} D_{R}^{c}-h_{e} A_{e} L H_{d} E_{R}^{c}+\text { h.c. }\right) \\
& +M_{1} \widetilde{B} \widetilde{B}+M_{2} \widetilde{W} \widetilde{W}+M_{3} \widetilde{g} \widetilde{g} .
\end{aligned}
$$

In this paper we study the general nMSSM with arbitrary soft terms at the SUSY scale as well as the semi-universal nMSSM for which one imposes the following constraints on the soft terms at the GUT scale

$$
\left\{\begin{array}{l}
m_{Q}=m_{U}=m_{D}=m_{L}=m_{E} \equiv m_{0} \\
A_{u}=A_{d}=A_{e} \equiv A_{0} \\
M_{1}=M_{2}=M_{3} \equiv M_{1 / 2} .
\end{array}\right.
$$

In both cases one can trade the (free) parameters $m_{H_{u}}, m_{H_{d}}, m_{S}$ for the Higgs VEVs $v_{u}, v_{d}, s$, or equivalently for $\mu \equiv \lambda s, \tan \beta \equiv \frac{v_{u}}{v_{d}}$ and the known value of $M_{Z}^{2}=g^{2} v^{2}$, where $g^{2}=\left(g_{1}^{2}+g_{2}^{2}\right) / 2, v=\sqrt{v_{u}^{2}+v_{d}^{2}} \approx 174 \mathrm{GeV}$, and $g_{1}, g_{2}$ denote the $\mathrm{U}(1)_{Y}$ and $\mathrm{SU}(2)_{L}$ gauge couplings, respectively. 
From the SUSY $F$ - and $D$-terms and the soft SUSY breaking terms one obtains the potential for the neutral Higgs fields:

$$
\begin{aligned}
V_{\text {Higgs }}= & \left|-\lambda H_{u}^{0} H_{d}^{0}+\xi_{F}\right|^{2}+\frac{g^{2}}{4}\left(\left|H_{u}^{0}\right|^{2}-\left|H_{d}^{0}\right|^{2}\right)^{2}+m_{S}^{2}|S|^{2} \\
& +\left(m_{H_{u}}^{2}+|\lambda S|^{2}\right)\left|H_{u}^{0}\right|^{2}+\left(m_{H_{d}}^{2}+|\lambda S|^{2}\right)\left|H_{d}^{0}\right|^{2} \\
& +\left(-\lambda A_{\lambda} H_{u}^{0} H_{d}^{0} S+\xi_{S} S+\text { h.c. }\right)
\end{aligned}
$$

which at the minimum is

$$
\begin{aligned}
V_{0}= & \left(-\lambda v_{u} v_{d}+\xi_{F}\right)^{2}+\frac{g^{2}}{4}\left(v_{u}^{2}-v_{d}^{2}\right)^{2}+m_{S}^{2} s^{2} \\
& +\left(m_{H_{u}}^{2}+\mu^{2}\right) v_{u}^{2}+\left(m_{H_{d}}^{2}+\mu^{2}\right) v_{d}^{2}-2 \lambda A_{\lambda} v_{u} v_{d} s+2 \xi_{S} s
\end{aligned}
$$

The minimisation equations are given by

$$
\left\{\begin{array}{l}
v_{u}\left(m_{H_{u}}^{2}+\mu^{2}+\lambda^{2} v_{d}^{2}+\frac{g 2}{2}\left(v_{u}^{2}-v_{d}^{2}\right)\right)-v_{d}\left(\mu A_{\lambda}+\lambda \xi_{F}\right)=0 \\
v_{d}\left(m_{H_{d}}^{2}+\mu^{2}+\lambda^{2} v_{u}^{2}+\frac{g 2}{2}\left(v_{d}^{2}-v_{u}^{2}\right)\right)-v_{u}\left(\mu A_{\lambda}+\lambda \xi_{F}\right)=0 \\
s\left(m_{S}^{2}+\lambda^{2}\left(v_{u}^{2}+v_{d}^{2}\right)\right)+\xi_{S}-\lambda A_{\lambda} v_{u} v_{d}=0
\end{array}\right.
$$

From the first two of these equations one can derive

$$
\frac{v_{u} v_{d}}{v^{2}} \equiv \frac{1}{2} \sin 2 \beta=\frac{\mu A_{\lambda}+\lambda \xi_{F}}{m_{H_{u}}^{2}+m_{H_{d}}^{2}+2 \mu^{2}+\lambda^{2} v^{2}},
$$

and from the third, one obtains in the limit where $s \gg v$ (or equivalently $\lambda \ll 1$ )

$$
\mu \simeq-\frac{\lambda \xi_{S}}{m_{S}^{2}}
$$

In the basis $\left(H_{d R}, H_{u R}, S_{R}\right)$ and after the elimination of $m_{H_{d}}^{2}, m_{H_{u}}^{2}$ and $m_{S}^{2}$ using the minimisation equations (2.12), the elements of the $3 \times 3 \mathrm{CP}$-even mass matrix $\mathcal{M}_{S}^{2}$ read

$$
\begin{aligned}
\mathcal{M}_{S, 11}^{2} & =g^{2} v_{d}^{2}+\left(\mu A_{\lambda}+\lambda \xi_{F}\right) \tan \beta \\
\mathcal{M}_{S, 22}^{2} & =g^{2} v_{u}^{2}+\left(\mu A_{\lambda}+\lambda \xi_{F}\right) / \tan \beta \\
\mathcal{M}_{S, 33}^{2} & =\frac{\lambda^{2} A_{\lambda} v_{u} v_{d}-\lambda \xi_{S}}{\mu} \\
\mathcal{M}_{S, 12}^{2} & =\left(2 \lambda^{2}-g^{2}\right) v_{u} v_{d}-\left(\mu A_{\lambda}+\lambda \xi_{F}\right), \\
\mathcal{M}_{S, 13}^{2} & =\lambda\left(2 \mu v_{d}-A_{\lambda} v_{u}\right) \\
\mathcal{M}_{S, 13}^{2} & =\lambda\left(2 \mu v_{u}-A_{\lambda} v_{d}\right) .
\end{aligned}
$$

Dropping the Goldstone mode, one can express the $2 \times 2 \mathrm{CP}$-odd mass matrix $\mathcal{P}_{S}^{2}$ in the basis $\left(A, S_{I}\right)$, where $A=\cos \beta H_{u I}+\sin \beta H_{d I}$

$$
\mathcal{M}_{P, 11}^{2}=\frac{2\left(\mu A_{\lambda}+\lambda \xi_{F}\right)}{\sin 2 \beta}, \quad \mathcal{M}_{P, 22}^{2}=\frac{\lambda^{2} A_{\lambda} v_{u} v_{d}-\lambda \xi_{S}}{\mu}, \quad \mathcal{M}_{P, 12}^{2}=\lambda A_{\lambda} v
$$


One can notice that $\mathcal{M}_{S, 33}^{2}=\mathcal{M}_{P, 22}^{2}$, i.e. in the limit of small mixing between the singlet and doublet sectors, the CP-even and CP-odd singlet states have the same mass (up to radiative corrections, see below). In addition, this common (tree level) mass depends on the tadpole parameter $\xi_{S}$ which is a free parameter. Hence singlet like Higgs masses are arbitrary. In particular, they can be lighter than $125 \mathrm{GeV}$ and still not excluded if their reduced couplings to SM particles (especially gauge bosons) are sufficiently suppressed.

Finally, in the basis $\psi^{0}=\left(-i \lambda_{1},-i \lambda_{2}^{3}, \psi_{d}^{0}, \psi_{u}^{0}, \psi_{S}\right)$, the neutralino mass matrix reads

$$
\mathcal{M}_{0}=\left(\begin{array}{ccccc}
M_{1} & 0 & -\frac{g_{1} v_{d}}{\sqrt{2}} & \frac{g_{1} v_{u}}{\sqrt{2}} & 0 \\
& M_{2} & \frac{g_{2} v_{d}}{\sqrt{2}} & -\frac{g_{2} v_{u}}{\sqrt{2}} & 0 \\
& & 0 & -\mu & -\lambda v_{u} \\
& & & 0 & -\lambda v_{d} \\
& & & & 0
\end{array}\right) .
$$

Therefore, in the limit of small singlino-higgsino mixing $(\lambda \ll 1)$, the singlino mass is $m_{\widetilde{S}}=0$. On the other hand, if either $\mu$ or $M_{1}$ and $M_{2}$ are much larger than $M_{Z}$, one gets [54]

$$
m_{\widetilde{S}} \simeq \frac{\mu \lambda^{2} v^{2}}{\mu^{2}+\lambda^{2} v^{2}} \sin 2 \beta .
$$

The experimental lower bound on $\mu$ (from the non observation of a light chargino) and the theoretical upper bound on $\lambda$ (assuming perturbativity up to the GUT scale) therefore yield an upper bound on the singlino mass $m_{\widetilde{S}} \lesssim 75 \mathrm{GeV}$.

The physical CP-even Higgs states will be denoted as $h_{i}, i=1,2,3$ (ordered in mass), and the physical CP-odd Higgs states as $a_{i}, i=1,2$. The neutralinos are denoted as $\tilde{\chi}_{i}^{0}$, $i=1 \ldots 5$ and their mixing angles $N_{i, j}$ such that $N_{1,5}$ indicates the singlino component of the lightest neutralino $\tilde{\chi}_{1}^{0}$ (assumed to be the LSP).

All the above expressions are for tree level mass matrices. Loop corrections play an important role, especially in the Higgs sector where they account for a large part of the SM like Higgs mass at $125 \mathrm{GeV}$. To compute the SUSY and Higgs spectrum, we have used the NMSSMTools package, setting the precision for radiative corrections to the minimum (precision flag for Higgs calculations $=0$ in the NMSSMTools input files). This includes the full one loop and the leading log two loop contributions from (s)top/(s)bottom, as well as the leading log one loop EW corrections. We have not used the most precise computation (precision flag for Higgs calculations $=2$ ) of ref. [80], as it is valid only for the $Z_{3}$ invariant NMSSM. In this ( $Z_{3}$ invariant) limit however, we have checked that the difference between the two computations is usually $\lesssim 3 \mathrm{GeV}$ for the SM like Higgs state near $125 \mathrm{GeV}$. In addition, a slight change of input parameters can always reproduce the same Higgs spectrum with both flags. ${ }^{2}$ The minimal precision for radiative corrections presents the extra advantage of using less CPU time, which is crucial for scans on large parameter space. In addition it allows to compute easily the complete effective Lagrangian in the Higgs sector with the same level of approximation. This complete Lagrangian can then be fed into micrOMEGAs so as to compute the relic density of the LSP DM candidate

\footnotetext{
${ }^{2}$ For a review of NMSSM Higgs mass calculations in public codes (including NMSSMTools), see ref. [81].
} 
as well as its DD and ID rates. Note that the higher-order corrections to the Higgs selfcouplings encoded in the effective Lagrangian can in some cases have a significant effect on the DM relic density.

\section{Parameter scan}

The parameter exploration of the semi-universal nMSSM (as defined in section 2) has been carried out using NMSSMTools v4.6.0, scanning over the following parameters: ${ }^{3}$

$$
m_{0}, M_{1 / 2}, A_{0}, \mu, \tan \beta, \lambda, \xi_{F}, \xi_{S}, A_{\lambda},
$$

which are all defined at the GUT scale except $\tan \beta$ (at $M_{Z}$ ) and $\lambda, \mu$ (at the SUSY scale). To efficiently scan over the nMSSM parameter space we have employed the Markov Chain Monte Carlo (MCMC) routines implemented in the NMSSMTools package, which we have tuned in order to cover in details regions of parameter space corresponding to lighter sparticles, i.e. with higher experimental prospects. Scenarios with very heavy sparticles (out of the LHC Run-2 reach) have been discarded.

We have applied all the default constraints implemented into NMSSMTools (except for the constraint on the anomalous magnetic moment of the muon $g_{\mu}-2$ ), which include in particular: ${ }^{4}$

- no unphysical minimum of the Higgs potential,

- no Landau pole below the GUT scale,

- invisible $\mathrm{Z}$ width $\Gamma_{Z}<0.5 \mathrm{MeV}$,

- B-physics constraints,

- LEP and Tevatron searches for sparticles and Higgs bosons,

- Tevatron and LHC searches on charged Higgs via top decays,

- at least one Higgs boson in the $125.1 \pm 3 \mathrm{GeV}$ mass range,

- $\chi^{2}$ fit to the Higgs signal strengths [82].

The latter indirectly takes into account the limit on non-standard decays of the SM like Higgs, such as the decay into light Higgs states or the invisible decays into the LSP which are somewhat dependent on shifts of other Higgs couplings. Moreover we have checked a posteriori that the direct limits on heavy Higgs states in the $W W$ channel were satisfied [83].

In performing our scan we have also required the DM relic density $\Omega h^{2}$ to be compatible with the relic abundance measured by Planck [84], $\Omega h_{\text {Planck }}^{2}=0.1186 \pm 0.0020$ at $68 \%$ CL. We have chosen to impose just an upper bound of the relic density, $\Omega h^{2}<0.131$, which takes

\footnotetext{
${ }^{3}$ The value of the top quark pole mass has been fixed to $m_{\text {top }}=173.1 \mathrm{GeV}$.

${ }^{4}$ See http://www.th.u-psud.fr/NMHDECAY/nmssmtools.html for a detailed list of the implemented constraints.
} 


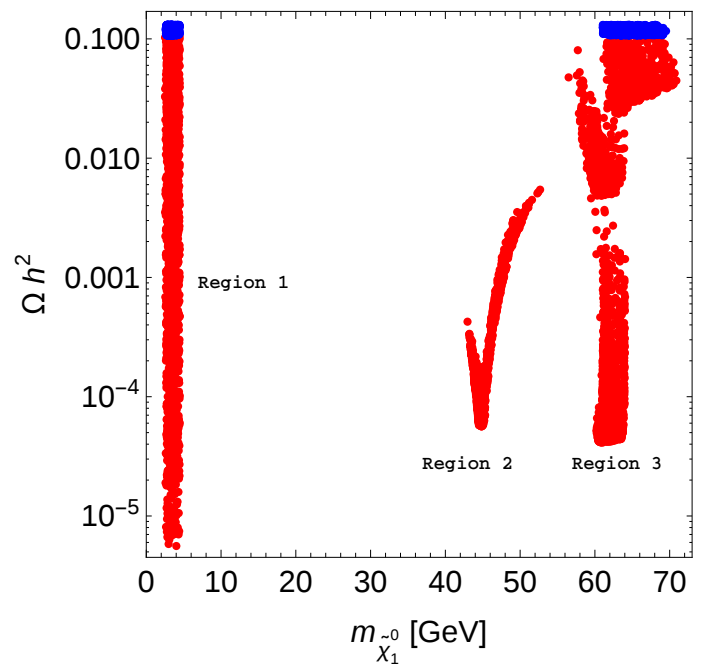

Figure 1. DM relic density $\Omega h^{2}$ in function of the LSP mass. Blue (red) points correspond to a DM relic density $0.107 \leq \Omega h^{2} \leq 0.131\left(\Omega h^{2}<0.107\right)$.

into account $\sim 10 \%$ theoretical uncertainties that could arise from loop corrections into the DM annihilation cross section, see e.g. [85]. We have also required the spin independent cross section for DD, rescaled for the local DM abundance $\left(\sigma_{\text {rescaled }}^{\mathrm{SI}}=\sigma^{\mathrm{SI}} \Omega h^{2} / \Omega h_{\text {Planck }}^{2}\right)$, to be compatible with the latest LUX results [86].

We illustrate in figure 1 the results of the scan mapped in the $m_{\tilde{\chi}_{1}^{0}}-\Omega h^{2}$ plane, showing in blue the points with a DM relic density compatible with Planck $\left(0.107<\Omega h^{2}<0.131\right)$ and in red the points for which it is below $\left(\Omega h^{2}<0.107\right)$. We see that three different regimes for the LSP mass exist: a region with a very light LSP below $5 \mathrm{GeV}$ (region 1), a region with $\mathrm{a} \sim 45 \mathrm{GeV} \operatorname{LSP}$ (region 2 ) and a region with a $\sim 65 \mathrm{GeV}$ LSP (region 3). In the first region the DM annihilation proceeds through a light pseudoscalar (singlet like) Higgs resonance, the second corresponds to the $Z$ resonance and the third to the exchange of a SM like Higgs or $Z$ boson. Note that the gap for neutralino masses between 5 and $40 \mathrm{GeV}$ is mostly due to constraints from the invisible width of the Higgs. The light LSP is a nearly pure singlino, so that the Higgs invisible width is very small. As the singlino mass increases, the same does its higgsino component, hence increasing the contribution to the Higgs invisible width.

As we will discuss in the following, the $5 \mathrm{GeV}$ and $65 \mathrm{GeV}$ LSP region present two and three sub-regions respectively, mapped in different areas of the $m_{0}-M_{1 / 2}$ parameter space. We report in tables 1-2 the maximum and minimum values for all of the nMSSM input parameters, in the three regions and sub-regions that we have identified. Moreover in this tables we also specify the weak scale gaugino masses and give a quick overview of the sparticle spectrum in the different sub-regions. All regions have small $\mu$ and several sub-regions feature sfermions and/or gauginos at the multi $\mathrm{TeV}$ scale. We distinguish region 1 for which the $125 \mathrm{GeV}$ (SM like) Higgs state is the second lightest CP even $\left(h_{2}\right)$ while the lightest $\left(h_{1}\right)$ is mainly singlet, from regions 2 and 3 where the lightest CP even Higgs state $\left(h_{1}\right)$ is SM like with a mass of $125 \mathrm{GeV}$. In the former case (with intermediate 


\begin{tabular}{|c|c|c|c|c|c|c|}
\hline Region & \multicolumn{2}{|c|}{$1 \mathrm{~A}$} & \multicolumn{2}{|c|}{$1 \mathrm{~B}$} & \multicolumn{2}{|c|}{2} \\
\hline $\tan \beta$ & 6.6 & 10 & 6 & 8 & 1.5 & 2.1 \\
\hline$\lambda$ & 0.33 & 0.53 & 0.49 & 0.52 & 0.68 & 0.80 \\
\hline$\mu$ & 240 & 400 & 350 & 430 & 115 & 180 \\
\hline$m_{0}$ & 0 & 1080 & 4040 & 4800 & $2 \cdot 10^{4}$ & $6 \cdot 10^{5}$ \\
\hline$M_{1 / 2}$ & 630 & 1200 & 280 & 440 & 180 & 470 \\
\hline$A_{0}$ & -1700 & 50 & 6700 & 7900 & $-3.7 \cdot 10^{4}$ & $-2.5 \cdot 10^{3}$ \\
\hline$A_{\lambda}$ & 1400 & 6000 & 7000 & 7900 & $-1.3 \cdot 10^{4}$ & $3.3 \cdot 10^{4}$ \\
\hline$\xi_{F}$ & 10 & 100 & $-1.5 \cdot 10^{4}$ & $-1.4 \cdot 10^{4}$ & $3.7 \cdot 10^{4}$ & $5.1 \cdot 10^{6}$ \\
\hline$\xi_{S}$ & $-6 \cdot 10^{4}$ & $2 \cdot 10^{4}$ & $-1.9 \cdot 10^{7}$ & $-1.6 \cdot 10^{7}$ & $-5.2 \cdot 10^{10}$ & $9.7 \cdot 10^{8}$ \\
\hline$M_{1}$ & 270 & 520 & 110 & 190 & 95 & 225 \\
\hline$M_{2}$ & 500 & 950 & 200 & 340 & 160 & 400 \\
\hline$m_{\tilde{q}}$ & 1300 & 2400 & \multicolumn{2}{|c|}{$>3000$} & \multicolumn{2}{|c|}{$>20000$} \\
\hline$m_{\tilde{t}_{1}}$ & 350 & 1300 & 1050 & 1900 & \multicolumn{2}{|c|}{$>3000$} \\
\hline$m_{\tilde{l}}$ & 180 & 1100 & \multicolumn{2}{|c|}{$>3000$} & \multicolumn{2}{|c|}{$>20000$} \\
\hline$m_{\tilde{g}}$ & 1450 & 2600 & 780 & 1250 & 800 & 1500 \\
\hline
\end{tabular}

Table 1. Maximum and minimum parameter values surviving the constraints implemented in NMSSMTools for regions 1 and 2. Also indicated are the $M_{1}$ and $M_{2}$ values at the SUSY scale and the mass ranges for sparticles. Dimensionfull parameters are expressed in $\mathrm{GeV}$ except $\xi_{F}$ and $\xi_{S}$ which are in $\mathrm{GeV}^{2}$ and $\mathrm{GeV}^{3}$ respectively.

values of $\tan \beta$ ) the SM like Higgs state takes its (relatively heavy) mass from $h_{1} / h_{2}$ mixing effects. In the latter, $\tan \beta \approx 2$ and $\lambda$ is near its maximal theoretically allowed value (from perturbativity up to the GUT scale). Hence the pure nMSSM contribution to the lightest Higgs mass is maximal.

In the next section we will analyse these three regions separately, first checking what are the constraints set by LHC Run-1 and ID experiments, then describing the prospects of this scenarios for the LHC Run-2, together with the prospects for future DD experiments.

To check the compatibility with the LHC constraints we have used both the packages SModelS v1.0.2 and MadAnalysis5. The former is a tool designed to decompose the signal of a given SUSY model into simplified topologies that are searched for by ATLAS and CMS, taking into account that in a generic SUSY spectrum the assumptions on the sparticles decays can be (and usually are) different from the ones assumed in the experimental searches. The input of SModelS consists of an SLHA file [87], containing particle spectrum and decay tables, together with SUSY production cross sections, which we have computed with micrOMEGAs v4.1.7. For strong production of sparticles we have also applied next-to-leading-order and next-to-next-to-leading-log (NLO+NNLL) QCD $\kappa$-factors, which have been computed with the nllfast package [88-94]. Note that due to the presence of many states below the TeV scale, and in particular of a very light LSP, for some regions of parameter space the coloured sparticles possess a large number of possible decay chains, including long ones. Hence building the list of existing topologies can be much 


\begin{tabular}{|c|c|c|c|c|c|c|}
\hline Region & \multicolumn{2}{|c|}{$3 \mathrm{~A}$} & \multicolumn{2}{|c|}{$3 \mathrm{~B}$} & \multicolumn{2}{|c|}{$3 \mathrm{C}$} \\
\hline $\tan \beta$ & 1.8 & 2.5 & 1.8 & 2.8 & 1.3 & 1.8 \\
\hline$\lambda$ & 0.64 & 0.77 & 0.66 & 0.74 & 0.65 & 0.74 \\
\hline$\mu$ & -140 & -90 & -110 & -90 & 110 & 150 \\
\hline$m_{0}$ & $3.9 \cdot 10^{3}$ & $6.6 \cdot 10^{4}$ & 170 & 2500 & 0 & 3150 \\
\hline$M_{1 / 2}$ & 130 & 210 & 200 & 560 & $5.6 \cdot 10^{3}$ & $2.3 \cdot 10^{4}$ \\
\hline$A_{0}$ & 11 & $3.3 \cdot 10^{4}$ & 440 & 3600 & $-1.9 \cdot 10^{4}$ & $4.7 \cdot 10^{3}$ \\
\hline$A_{\lambda}$ & $6.2 \cdot 10^{3}$ & $2.7 \cdot 10^{4}$ & 450 & 3500 & 4 & 8200 \\
\hline$\xi_{F}$ & $4.8 \cdot 10^{5}$ & $3.4 \cdot 10^{7}$ & $4.3 \cdot 10^{5}$ & $7.5 \cdot 10^{6}$ & $4.6 \cdot 10^{5}$ & $1.8 \cdot 10^{7}$ \\
\hline$\xi_{S}$ & $2.0 \cdot 10^{9}$ & $1.3 \cdot 10^{11}$ & $1.0 \cdot 10^{8}$ & $4.9 \cdot 10^{9}$ & $-1.9 \cdot 10^{9}$ & $3.0 \cdot 10^{9}$ \\
\hline$M_{1}$ & 52 & 65 & 85 & 230 & $2.6 \cdot 10^{3}$ & $1.1 \cdot 10^{4}$ \\
\hline$M_{2}$ & 83 & 108 & 160 & 430 & $4.6 \cdot 10^{3}$ & $2.0 \cdot 10^{4}$ \\
\hline$m_{\tilde{q}}$ & \multicolumn{2}{|c|}{$>3000$} & 780 & 2500 & \multirow{2}{*}{\multicolumn{2}{|c|}{$\begin{array}{l}>10000 \\
>5000\end{array}$}} \\
\hline$m_{\tilde{t}_{1}}$ & 500 & $>20000$ & 150 & 550 & & \\
\hline$m_{\tilde{l}}$ & \multicolumn{2}{|c|}{$>3000$} & 100 & 2500 & 100 & 3000 \\
\hline$m_{\tilde{g}}$ & 450 & 650 & 590 & 1300 & \multicolumn{2}{|c|}{$>10000$} \\
\hline
\end{tabular}

Table 2. Maximum and minimum parameter values surviving the constraints implemented in NMSSMTools for region 3 . See caption of table 1 .

more computer-time consuming than in the MSSM. Conversely MadAnalysis5 is a multi purpose package designed for the analysis of events generated at parton level and/or reconstructed level with the inclusion of parton showering and detector effects. In this work we have exploited the MadAnalysis5 Public Analysis Database, i.e. a list of implemented and validated experimental searches, with which one can recast the experimental limits set by ATLAS and CMS in a generic model or in a particular model configuration. Clearly the approach of MadAnalysis5 is more general, since it does not rely on any simplified model assumption, but it has the drawback of being somewhat more time consuming, given the need to generate events with a Monte Carlo generator for each model point that one wants to test. In this paper we will use a combination of both tools to check the limits set by LHC Run-1 in the nMSSM, using MadAnalysis5 especially to set bounds on the gluino mass.

\section{LHC and DM phenomenology}

After having defined the different regions of the GUT scale parameter space obeying the basic set of collider, astrophysical, cosmological and theoretical constraints, we now examine for each scenario the constraints from searches for sparticles at LHC Run-1 as well as from indirect searches for DM. For each scenario we then discuss what are the most relevant searches at LHC Run-2, both for sparticles and Higgs states, and we provide benchmark points with specific nMSSM signatures. The complementarity with direct DM searches is also highlighted. 


\subsection{Region 1, $m_{\tilde{\chi}_{1}^{0}}<5 \mathrm{GeV}$}

In this region the LSP is a quasi pure singlino state and it can appear in two different configurations of the $m_{0}-M_{1 / 2}$ parameter space. One is characterised by small $m_{0}(\lesssim$ $1 \mathrm{TeV}$ ) and $M_{1 / 2} \sim 1 \mathrm{TeV}$ (region $1 \mathrm{~A}$ ) while the second one has small $M_{1 / 2}(\lesssim 500 \mathrm{GeV})$ and large $m_{0} \sim 4 \mathrm{TeV}$ (region 1B). These two regions give rise to different sparticle spectra that we will analyse separately. We however stress three common features in these subregions. The first is that the lightest pseudoscalar and the singlino masses are linked, with $\left|m_{a_{1}}-2 m_{\tilde{\chi}_{1}^{0}}\right| \lesssim 1.5 \mathrm{GeV}$, in order to ensure efficient annihilation of the singlino through $a_{1}$ resonance, so as to satisfy the relic density constraint. The second follows from the singlino nature of the LSP. Decays of sparticles to a final state containing the LSP will often be suppressed thus, phase space allowing, longer decay chains involving heavier neutralinos, such as $\tilde{t}_{1} \rightarrow t \tilde{\chi}_{2}^{0} \rightarrow t \tilde{\chi}_{1}^{0} Z$, will be preferred. This has important consequences for simplified models limits. Finally, the measured DM relic abundance can be accounted for just by the LSP itself. However, as explained in the previous section, we will work in the less constraining assumption of a relic abundance $\Omega h^{2}<0.131$, thus allowing for the possibility of another DM component.

\subsubsection{Current constraints}

In the region with small $m_{0}(1 \mathrm{~A})$ the spectrum is characterised by the presence of light stops $\left(m_{\tilde{t}_{1}}<1300 \mathrm{GeV}\right)$ and light sleptons $\left(m_{\tilde{l}} \sim 180-1100 \mathrm{GeV}\right)$, with first and second generation squarks heavier than $1.3 \mathrm{TeV}$ and gluinos heavier than $1.45 \mathrm{TeV}$. The most stringent constraints on coloured sparticles are thus easily evaded. Given the values of $\mu, M_{1}$ and $M_{2}$ reported in table 1, the EWino spectrum is characterised, besides the light singlino, by four neutral and charged states with a mass between 240 and $520 \mathrm{GeV}$ corresponding to the three higgsinos and the bino. In the Higgs sector the role of the SM Higgs boson is played by $h_{2}$, while the $h_{1}$ mass is between 35 and $70 \mathrm{GeV}$ and the lightest pseudoscalar $a_{1}$ has a mass $\sim 2 m_{\tilde{\chi}_{1}^{0}}$. The masses of the three states of the heavy Higgs doublet are above 1.5 TeV. Note that pure scalar and pseudoscalar singlets are expected to have the same mass, however the mixing with the light scalar doublet is sufficient to increase the mass of the scalar singlet by a few tens of $\mathrm{GeV}$.

We show in figure 2 the allowed and excluded points after the application of the constraints implemented in SModelS, projected in the $m_{\tilde{e}_{L}}-m_{\tilde{t}_{1}}$ plane. For each excluded point we indicate the most constraining analysis. This is defined in SModelS as the analysis that has the larger ratio between the theoretical prediction and the experimental measurement for a given channel. However more than one channel can exclude the same point. From figure 2 it is clear that the most constraining searches are the ATLAS search for slepton production [95] $\left(\tilde{l} \rightarrow l \tilde{\chi}_{1}^{0}\right)$ and the CMS search for stop production [96] $\left(\tilde{t} \rightarrow b \tilde{\chi}_{1}^{+}\right.$and $\left.\tilde{t} \rightarrow t \tilde{\chi}_{1}^{0}\right)$. In particular the former is able to exclude sleptons lighter than $\sim 300 \mathrm{GeV}$ while the latter sets a lower bound of $\sim 550 \mathrm{GeV}$ on the lightest stop mass. These are stringent bounds, in the sense that no sparticles lighter than these limits are allowed. It is important to notice that, while the reach on the slepton mass is close to the official result of the ATLAS analysis for a $5 \mathrm{GeV}$ LSP $\left(m_{\tilde{l}} \gtrsim 330 \mathrm{GeV}\right)$, this is not the case for the stop 


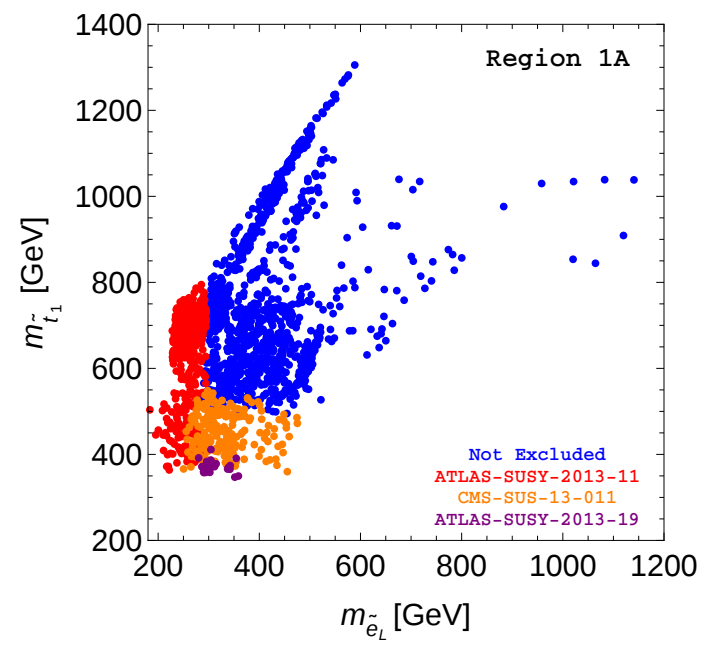

Figure 2. Allowed and excluded points for region 1A in the $m_{\tilde{e}_{L}}-m_{\tilde{t}_{1}}$ plane. For each excluded point we indicate the search with the maximum sensitivity.

search, where the ATLAS limit is around $650 \mathrm{GeV}$. However this can be explained by the fact that the simplified model result assumes a $100 \%$ branching ratio either for $\tilde{t}_{1} \rightarrow t \tilde{\chi}_{1}^{0}$ or $\tilde{t}_{1} \rightarrow b \tilde{\chi}_{1}^{+}$, an assumption which is not satisfied here. First, the mass spectrum is such that there is always at least one decay channel into a heavier neutralino which is allowed. Moreover, the decays into heavier neutralinos typically have larger branching ratios than the decay into the singlino LSP. This causes therefore a small reduction of the LHC exclusion reach.

The second region with a light LSP (1B) corresponds to large $m_{0}(\sim 4 \mathrm{TeV})$ and small $M_{1 / 2}$. The large value of $m_{0}$ yields heavy sfermions with slepton and squark masses above $3 \mathrm{TeV}$, except for the lightest stop which is between $1-2 \mathrm{TeV}$. Therefore the only sub-TeV sparticles are EWinos and gluinos. In particular the neutralino spectrum has the following hierarchy: a light singlino $\left(m_{\tilde{\chi}_{1}^{0}}<5 \mathrm{GeV}\right)$, a bino $\left(m_{\tilde{\chi}_{2}^{0}} \sim 100-200 \mathrm{GeV}\right)$, a wino $\left(m_{\tilde{\chi}_{3}^{0}} \sim 200-300 \mathrm{GeV}\right)$ which is degenerate with the lightest chargino, and heavier higgsino states since typically $\mu$ is larger than $M_{1}, M_{2}$ in region $1 \mathrm{~A}$ (see table 1 ). The gluinos lie in the $800-1200 \mathrm{GeV}$ range, while $h_{1}$ is heavier than in region $1 \mathrm{~A}$, between 70 and $90 \mathrm{GeV}$.

Contrary to what we found in region $1 \mathrm{~A}$, SModels does not set any constraint on the parameter space of this region. Clearly sleptons and squarks, including stops, escape the limits set by the experimental searches due to their high masses. Conversely, the reasons for EWinos escaping the LHC limits are less straightforward. The chargino mass in region $1 \mathrm{~B}$ is typically above the bound set by ATLAS from the search for $\tilde{\chi}_{1}^{+} \tilde{\chi}_{1}^{-}$production, with a subsequent $\tilde{\chi}_{1}^{ \pm} \rightarrow W^{ \pm} \tilde{\chi}_{1}^{0}$ decay, which, for a light LSP, is $m_{\tilde{\chi}^{+}}>180 \mathrm{GeV}$ [95]. The other simplified topology analysed by ATLAS which is relevant for region $1 \mathrm{~B}$ is $\tilde{\chi}_{1}^{ \pm} \tilde{\chi}_{2}^{0} \rightarrow W Z \tilde{\chi}_{1}^{0} \tilde{\chi}_{1}^{0}$. It assumes 100\% EWinos decays into the LSP plus a SM gauge boson, as well as pure wino states $\left(m_{\tilde{\chi}_{1}^{+}}=m_{\tilde{\chi}_{2}^{0}}\right)$. Considering the neutralino compositions, the latter criteria applies in our case only to $\tilde{\chi}_{3}^{0}$ while the decay assumption applies only to $\tilde{\chi}_{1}^{ \pm}$. Indeed, the branching ratio for $\tilde{\chi}_{3}^{0} \rightarrow Z \tilde{\chi}_{1}^{0}$ can be suppressed when decay rates into $h_{1,2} \tilde{\chi}_{1}^{0}$ and $a_{1} \tilde{\chi}_{1}^{0}$ become 
important. The same comment can be made about the $\tilde{\chi}_{2}^{0}$ decays. Moreover the $\tilde{\chi}_{2}^{0}$ composition, which is mainly bino, causes a significant decrease of the $\tilde{\chi}_{1}^{ \pm} \chi_{2}^{0}$ cross sections with respect to the wino case assumed in simplified models. Finally, the lack of sensitivity of the ATLAS search in a $3 l+E_{T}^{\text {miss }}$ final state [97] can be explained by the small branching ratios of the neutralinos in lepton pairs and LSP, which is typical of scenarios with heavy sleptons.

The case of the gluino deserves special consideration, since a priori its mass can be well within the range excluded by simplified models analyses. As before, the issue is that we find reduced branching ratios in the standard search channels $\left(\tilde{g} \rightarrow t \bar{t} \tilde{\chi}_{1}^{0}, t \bar{b} \tilde{\chi}_{1}^{0}, q \bar{q} \tilde{\chi}_{1}^{0}\right)$ because of competing decays into heavier neutral and charged EWinos, that can once again be linked to the singlino nature of the LSP. However, gluino searches generally rely on a large number of jets in the final state. These signal regions can be easily fed also from gluino decays different from the standard assumptions, in particular decays into heavier neutralinos.

We have then used the recast [98] CMS gluino search [99], implemented in the package MadAnalysis5, which relies on an opposite sign dilepton final state, more than 4 jets and more than 2 b-tag jets, together with a large $E_{T}^{\text {miss }}$. This search sets a $95 \%$ CL exclusion when more than 4 events fall in its unique signal region.

We have simulated gluino pair production with MadGraph5 v1.5.11 [100]. Parton showering, hadronization and decay of unstable particles have been performed with the package Pythia v6.4 [101], while the MadAnalysis5 tuned version of Delphes v3.2.0 [102] has been used to simulate detector effects. Jets are reconstructed with FastJet [103], via an anti- $k_{T}$ [104] algorithm. We have then computed, through the efficiency map function implemented in MadAnalysis5, acceptances times efficiencies for various values of the gluino mass. From these, we have then calculated the final number of events for our nMSSM scenarios, using the production cross sections for gluino pair production computed via micrOMEGAs and nllfast. We have found a lower limit on the gluino mass of $\sim 1.1 \mathrm{TeV}$, close to the official CMS result [99]. This result strongly constrains region 1B, leaving just a few points with a heavy enough gluino.

Finally, we have also computed the ID cross section for LSP pair annihilation into $\tau^{+} \tau^{-}$final state (the most relevant at these masses) rescaled by the square of the local DM density, $\left[\Omega h^{2} /\left(\Omega h^{2}\right)_{\text {Planck }}\right]^{2}$, and compared it with the exclusion limits set by FermiLAT [105]. The results are shown in figure 3. For a $\sim 5 \mathrm{GeV}$ LSP the Fermi-LAT limit is approximately constant and equal to $10^{-27} \mathrm{~cm}^{3} / \mathrm{s}$. In the left panel we show all the points surviving the LHC Run-1 constraints (implemented as discussed above), while in the right panel we zoom on to the region with small $m_{a_{1}}-2 m_{\tilde{\chi}_{1}^{0}}$. Blue (red) points correspond to a DM relic density $0.107 \leq \Omega h^{2} \leq 0.131\left(\Omega h^{2}<0.107\right)$. From the plots it is clear that the current results from Fermi-LAT are able to completely exclude the portion of parameter space which corresponds to $m_{a_{1}}<2 m_{\tilde{\chi}_{1}^{0}}$, while above this threshold ID detection rates are well below the experimental limits. The reason for this is simply that when $2 m_{\tilde{\chi}_{1}^{0}}$ is just below $m_{a_{1}}$, its annihilation cross section at small velocities can be significantly enhanced by the resonance effect, while in the early universe the thermal velocity of the neutralino is enough to overshoot the resonance $[33,106]$. The increase in the rescaled cross section 

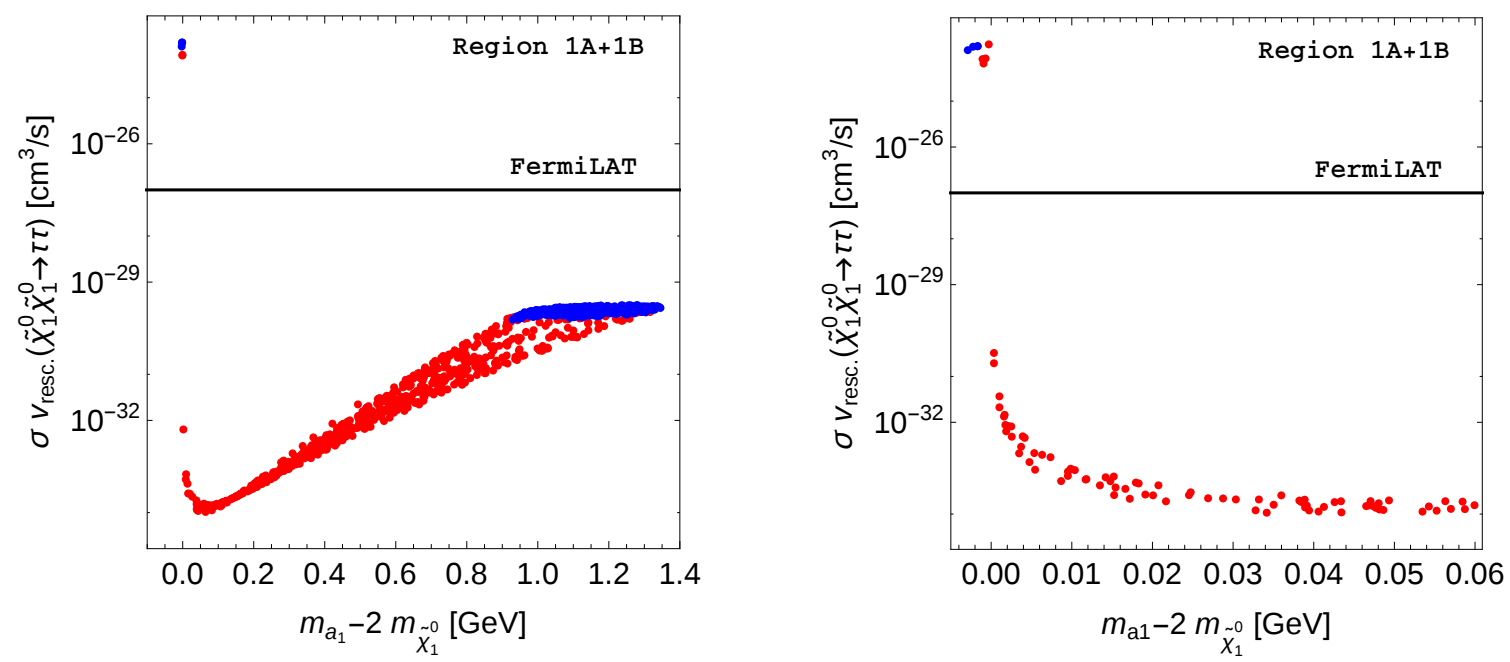

Figure 3. ID detection rates into $\tau^{+} \tau^{-}$final state in function of $m_{a_{1}}-2 m_{\tilde{\chi}_{1}^{0}}$. Blue (red) points correspond to a DM relic density $0.107 \leq \Omega h^{2} \leq 0.131\left(\Omega h^{2}<0.107\right)$. The horizontal lines represent the current limit from Fermi-LAT. Regions 1A and 1B are shown together.

with $m_{a_{1}}-2 m_{\tilde{\chi}_{1}^{0}}>0.1 \mathrm{GeV}$ in figure 3 (left panel) is an effect of the rescaling. Indeed $\sigma v$ in the galaxy as well as in the early universe decreases when moving away from the narrow pseudoscalar resonance until one reaches a region where $m_{a_{1}}-2 m_{\tilde{\chi}_{1}^{0}}$ is too large to benefit from a strong resonance enhancement and the relic density is in agreement with PLANCK data. However since the rescaling factor is inversely proportional to the square of the annihilation cross section in the early universe, the net effect is an increase of the rescaled cross section for DD until $m_{a_{1}}-2 m_{\tilde{\chi}_{1}^{0}} \approx 1 \mathrm{GeV}$. Note that limits from AMS antiproton are not expected to be important for such low masses [107].

\subsubsection{LHC Run-2 and direct detection experiments prospects}

We now discuss the prospects for the LHC Run-2, including sparticles and Higgs searches, as well as DD experiments for the points surviving the constraints imposed by the LHC Run-1 and ID measurements.

As shown in figure 2 standard searches for stops quarks are already quite effective in constraining light stops in region $1 \mathrm{~A}$, for which the dominant decay mode is $\tilde{t}_{1} \rightarrow b \tilde{\chi}_{1}^{+}$, and Run-2 is therefore expected to significantly extend the reach in this channel. The standard search channel $\tilde{t}_{1} \rightarrow t \tilde{\chi}_{1}^{0}$ does not offer as good prospects, since the branching ratio into the singlino LSP is typically suppressed. For example we found a $600 \mathrm{GeV}$ stop with a $\sim 60 \%$ branching ratio into a chargino and only $\sim 5 \%$ in $t \tilde{\chi}_{1}^{0}$, with the rest of the decays being saturated by $t \tilde{\chi}_{2,3}^{0}$. Since $\tilde{\chi}_{2,3}^{0}$ mainly decay into a $Z$ or a SM like Higgs boson and a LSP, final states with $t Z+E_{T}^{\text {miss }}$ or $t h_{2}+E_{T}^{\text {miss }}$, arising from one or both of the pair produced stops, provide a characteristic signature in this region. Also slepton searches will clearly act as a powerful probe of this region. In fact, only a factor two improvement in the LHC Run-2 mass reach, relative to the one of Run-1, would cover most of region 1A, leaving only a few points with sleptons heavier than $500 \mathrm{GeV}$. Upgrades of standard EWinos searches, 

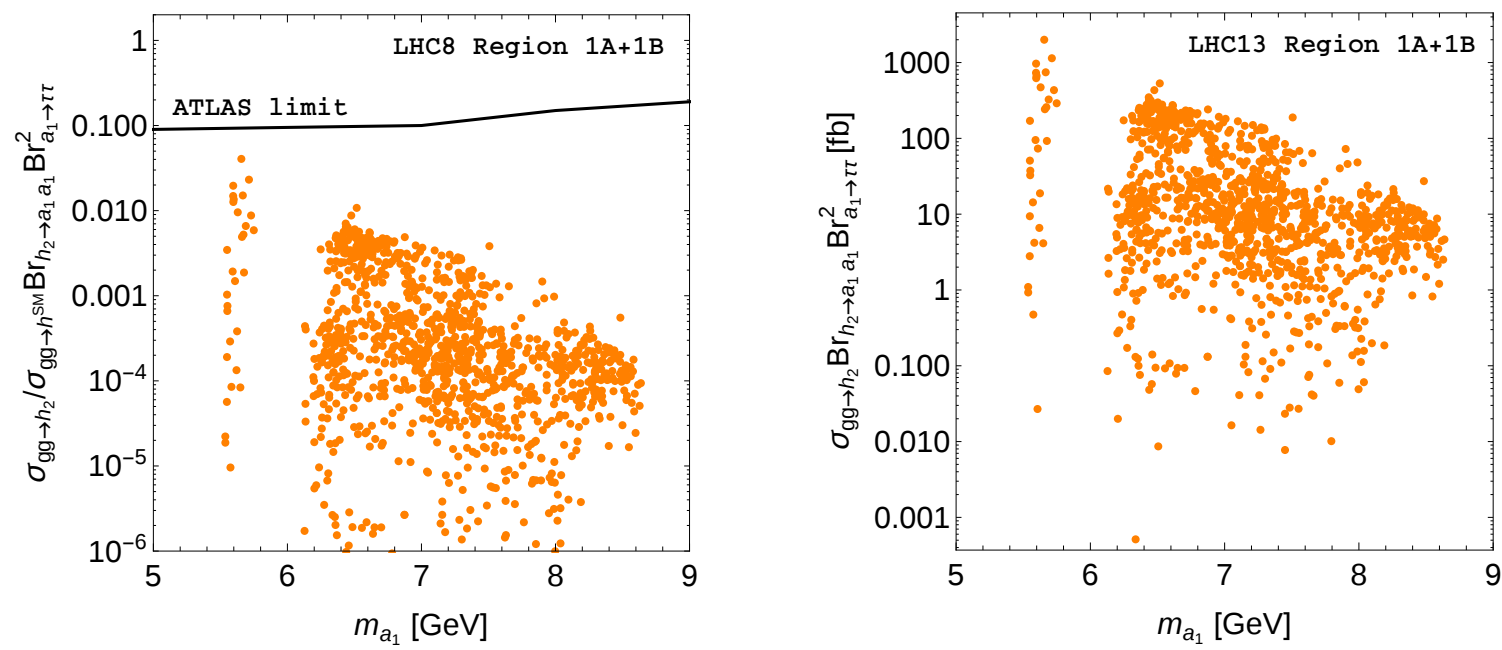

Figure 4. $\sigma\left(g g \rightarrow h_{2}\right) / \sigma\left(g g \rightarrow h^{\mathrm{SM}}\right) \operatorname{Br}\left(h_{2} \rightarrow a_{1} a_{1}\right) \mathrm{Br}^{2}\left(a_{1} \rightarrow \tau \tau\right)$ for the LHC Run-1 (left) and $\sigma\left(g g \rightarrow h_{2}\right) \operatorname{Br}\left(h_{2} \rightarrow a_{1} a_{1}\right) \mathrm{Br}^{2}\left(a_{1} \rightarrow \tau \tau\right)$ for the LHC Run-2 (right) for region $1 \mathrm{~A}$ and $1 \mathrm{~B}$. The horizontal line in the left plot is the limit set by ATLAS [108].

as in ref. [97], will also be able to further extend the LHC reach. In fact, even if in figure 2 the strongest bound represented is the one arising from slepton pair production, the decay pattern of $\tilde{\chi}_{1}^{+}$and $\tilde{\chi}_{2}^{0}$ can be consistent with the assumption in the experimental searches (e.g. $\tilde{\chi}_{2}^{0} \rightarrow \tilde{\chi}_{1}^{0} Z$ and $\tilde{\chi}_{1}^{ \pm} \rightarrow \tilde{\chi}_{1}^{0} W$ ), the latter especially if sleptons are heavier than EWinos. Conversely, for region $1 \mathrm{~B}$, gluino searches will clearly represent the major probe for LHC Run-2, since $m_{\tilde{g}}<1.2 \mathrm{TeV}$ and exclusion limits on the gluino mass are expected to greatly increase already with early $13 \mathrm{TeV}$ data.

The Higgs sector of these regions is characterised by a light pseudoscalar $a_{1}$, accompanied by a light scalar $h_{1}$, both with a dominant singlet component. The mass of $h_{1}$ lies between $30-70 \mathrm{GeV}$ in region $1 \mathrm{~A}$ and $70-90 \mathrm{GeV}$ in region $1 \mathrm{~B}$. This leads, in region $1 \mathrm{~A}$, to the interesting possibility of exotic decays of the $125 \mathrm{GeV}$ (SM like) Higgs state $h_{2}$ into a pair of light scalars $\left(h_{2} \rightarrow h_{1} h_{1}\right)$ as well as pseudoscalars $\left(h_{2} \rightarrow a_{1} a_{1}\right)$. Given the mass of the $a_{1}$ and of the $h_{1}$, possible interesting signatures to be explored are $h_{2} \rightarrow a_{1} a_{1} \rightarrow 4 \tau / 2 \tau 2 \mu$ and $h_{2} \rightarrow h_{1} h_{1} \rightarrow 4 b / 2 b 2 \tau$.

The leptonic channels have already been investigated at LHC Run-1. The ATLAS collaboration has performed a search for a CP even Higgs boson produced through gluon fusion and decaying into a pair of pseudoscalars, and has set a limit on the production cross section times branching ratio into a $4 \tau$ final state normalized to the SM Higgs boson cross section [108]. This limit can be easily related to the $2 \mu 2 \tau$ final state under the assumption that $\operatorname{Br}\left(a_{1} \rightarrow \mu \mu\right) / \operatorname{Br}\left(a_{1} \rightarrow \tau \tau\right)=m_{\mu}^{2} /\left(m_{\tau}^{2} \sqrt{1-\left(2 m_{\tau} / m_{a_{1}}\right)^{2}}\right)$. We show in figure 4 (left panel) the quantity $\sigma\left(g g \rightarrow h_{2}\right) / \sigma\left(g g \rightarrow h^{\mathrm{SM}}\right) \operatorname{Br}\left(h_{2} \rightarrow a_{1} a_{1}\right) \mathrm{Br}^{2}\left(a_{1} \rightarrow \tau \tau\right)$ for region $1 \mathrm{~A}$ and $1 \mathrm{~B}$, together with the limit set by the ATLAS collaboration. The rescaled production cross section has been computed using the reduced $g g h_{2}$ coupling provided by NMSSMTools, which is given with respect to a SM Higgs boson of the same mass. Given the small size of the deviations of the $h_{2}$ couplings with respect to the SM Higgs $\left(g_{g g h_{2}} / g_{g g h_{2}}^{\mathrm{SM}} \sim 1\right)$, and 
assuming $\sigma\left(g g \rightarrow h_{2}\right)_{\mathrm{SM}} \sim 20 \mathrm{pb}$ for $m_{h}^{\mathrm{SM}} \sim 125 \mathrm{GeV}$, this analysis sets a limit on the inclusive cross section times branching ratio of $\sim 2 \mathrm{pb}$ for $m_{a_{1}}=5-10 \mathrm{GeV}$. While this analysis does not constrain at the moment region 1 of the nMSSM, prospects for LHC Run-2 are quite exciting, given an inclusive cross section that can reach the $\sim$ pb level in the $a_{1}$ considered mass range (right panel). A recently published CMS analysis [109] also sets limits on the same production process, decaying however into a $4 \mu$ final state. In the case where the only source of signal events is given by $h_{2} \rightarrow a_{1} a_{1} \rightarrow 4 \mu$, the limit is set to $1 \mathrm{fb}$ for $m_{a_{1}}=3.55 \mathrm{GeV}$, which is well above the maximum rate obtained in the $\operatorname{nMSSM}(\sim 0.01 \mathrm{fb})$.

As mentioned, other interesting possibilities are the decays of the SM like Higgs boson into a pair of lighter CP even Higgs states $\left(h_{2} \rightarrow h_{1} h_{1}\right)$, which then decay into a $2 b 2 \tau$ final state for which rates could reach $\mathcal{O}(250 \mathrm{fb})$ at the $13 \mathrm{TeV}$ LHC. The decay into a $4 b$ final state has a larger rate, $\mathcal{O}(1 \mathrm{pb})$, but suffers from a higher QCD background [110]. While our discussion has been carried out assuming dominant gluon fusion production of the SM like Higgs boson, the contribution of different production modes (vector boson fusion or Higgs Strahlung) can improve the signal over background ratio, despite smaller production cross sections. Finally, direct production of a light pseudoscalar $a_{1}$, either via gluon fusion or via $b \bar{b}$ associate production, can provide other possible signatures for the search of a light pseudoscalar [110].

In region 1 the prospects for DM DD are quite pessimistic, due to the lightness of the LSP which has a mass below the lower limit that can be tested by the XENON1T experiment [111]. The value of the (rescaled) spin independent DD cross section, being at most $\sim 10^{-10} \mathrm{pb}$, is also problematic. This is about 1 order of magnitude below the neutrino coherent scattering background for a WIMP mass of $\sim 5 \mathrm{GeV}[112]$.

We conclude this section proposing in table 3 three benchmark points in region $1 \mathrm{~A}$, with the Higgs sector signatures relevant for the $13 \mathrm{TeV}$ run of the LHC discussed above. Table 3 reports also the relevant masses, cross sections (obtained with SusHi v1.5.0 [113, 114 ] at NNLO) and branching ratios for the various benchmark points. These scenarios are amenable for a deeper phenomenological investigation, both from the theoretical and experimental side. The benchmark points BMP1A-I and BMP1A-II are in a configuration where $m_{h_{2}}>2 m_{h_{1}}$, and maximise $\operatorname{Br}\left(h_{2} \rightarrow a_{1} a_{1}\right)$ and $\operatorname{Br}\left(h_{2} \rightarrow h_{1} h_{1}\right)$ respectively, with the possibility of giving rise to the aforementioned multi- $\tau$ or multi- $b$ final states, although the branching ratios are only $\sim 4 \%$ for $4 b$ and $\sim 1 \%$ for $2 b 2 \tau$. We also propose a third scenario, BMP1A-III, where the $h_{2} \rightarrow h_{1} h_{1}$ channel is kinematically closed, and the decay rate of the SM like Higgs in two light pseudoscalars is below $10^{-3}$. The decay pattern of $h_{1}$ and $a_{1}$ in this configuration are similar to the ones of BMP1A-I and BMP1A-II, but clearly these states now need to be produced directly. We do not propose benchmark points for region $1 \mathrm{~B}$ for two reasons. First, the characteristics of the Higgs sector are quite similar to the ones of region $1 \mathrm{~A}$ when the decay pattern $h_{2} \rightarrow h_{1} h_{1}$ is closed (recall in fact that in region $1 \mathrm{~B}$ the $h_{1}$ mass is between 70 and $90 \mathrm{GeV}$ ). Secondly, and more importantly, the lighter gluino in this region is likely to be tested with early data from LHC Run-2 through conventional search channels. 


\begin{tabular}{|l|c|c|c|}
\hline & BMP1A-I & BMP1A-II & BMP1A-III \\
\hline $\tan \beta$ & 7.8 & 8.32 & 9.48 \\
\hline$\lambda$ & 0.372 & 0.4 & 0.509 \\
\hline$\mu$ & 265 & 290 & 375 \\
\hline$m_{0}$ & 0 & 0 & 505 \\
\hline$M_{1 / 2}$ & 766 & 790 & 888 \\
\hline$A_{0}$ & -1146 & -1050 & 9 \\
\hline$A_{\lambda}$ & 2286 & 2700 & 5715 \\
\hline$\xi_{F}$ & 28 & 48 & 79 \\
\hline$\xi_{S}$ & $-5.3 \cdot 10^{4}$ & $-4.6 \cdot 10^{4}$ & 279 \\
\hline \hline $\operatorname{Masses}$ & $m_{h_{1}}=37.0 m_{a_{1}}=6.8$ & $m_{h_{1}}=43.6 m_{a_{1}}=6.8$ & $m_{h_{1}}=64.7 m_{a_{1}}=8.0$ \\
\hline$\sigma^{13 \mathrm{TeV}}[\mathrm{pb}]$ & $\sigma\left(g g \rightarrow h_{2}\right)=41.5$ & $\sigma\left(g g \rightarrow h_{2}\right)=42.2$ & $\sigma\left(g g \rightarrow h_{2}\right)=42.4$ \\
& $\sigma\left(g g \rightarrow h_{1}\right)=13.0$ & $\sigma\left(g g \rightarrow h_{1}\right)=1.8$ & $\sigma\left(g g \rightarrow h_{1}\right)=1.5$ \\
& $\sigma\left(g g \rightarrow a_{1}\right)=242.8$ & $\sigma\left(g g \rightarrow a_{1}\right)=236.5$ & $\sigma\left(g g \rightarrow a_{1}\right)=244.5$ \\
\hline $\operatorname{Br}\left(h_{2}\right)$ & $\operatorname{Br}\left(h_{2} \rightarrow a_{1} a_{1}\right)=8 \%$ & $\operatorname{Br}\left(h_{2} \rightarrow h_{1} h_{1}\right)=10 \%$ & \\
\hline $\operatorname{Br}\left(h_{1}\right)$ & $\operatorname{Br}\left(h_{1} \rightarrow b \bar{b}\right)=85 \%$ & $\operatorname{Br}\left(h_{1} \rightarrow b \bar{b}\right)=65 \%$ & $\operatorname{Br}\left(h_{1} \rightarrow b \bar{b}\right)=58 \%$ \\
& $\operatorname{Br}\left(h_{1} \rightarrow \tau \tau\right)=7 \%$ & $\operatorname{Br}\left(h_{1} \rightarrow a_{1} a_{1}\right)=28 \%$ & $\operatorname{Br}\left(h_{1} \rightarrow a_{1} a_{1}\right)=33 \%$ \\
& $\operatorname{Br}\left(h_{1} \rightarrow \tilde{\chi}_{1}^{0} \tilde{\chi}_{1}^{0}\right)=7 \%$ & $\operatorname{Br}\left(h_{1} \rightarrow \tau \tau\right)=6 \%$ & $\operatorname{Br}\left(h_{1} \rightarrow \tau \tau\right)=5 \%$ \\
\hline $\operatorname{Br}\left(a_{1}\right)$ & $\operatorname{Br}\left(a_{1} \rightarrow \tilde{\chi}_{1}^{0} \tilde{\chi}_{1}^{0}\right)=73 \%$ & $\operatorname{Br}\left(a_{1} \rightarrow \tilde{\chi}_{1}^{0} \tilde{\chi}_{1}^{0}\right)=73 \%$ & $\operatorname{Br}\left(a_{1} \rightarrow \tilde{\chi}_{1}^{0} \tilde{\chi}_{1}^{0}\right)=78 \%$ \\
& $\operatorname{Br}\left(a_{1} \rightarrow \tau \tau\right)=25 \%$ & $\operatorname{Br}\left(a_{1} \rightarrow \tau \tau\right)=25 \%$ & $\operatorname{Br}\left(a_{1} \rightarrow \tau \tau\right)=20 \%$ \\
\hline
\end{tabular}

Table 3. Benchmark points choices for region 1A. Dimensionful parameter are expressed in GeV, except $\xi_{F}$ and $\xi_{S}$ which are in $\mathrm{GeV}^{2}$ and $\mathrm{GeV}^{3}$ respectively and $\sigma$, expressed in pb. We indicate in the table the relevant mass spectrum, cross sections and branching ratios.

In summary, the Run-2 of the LHC at higher energy and luminosity will further probe region 1 through stop, slepton and gluino searches, while new Higgs decay channels involving light pseudoscalar and/or scalar bosons can provide characteristic signatures of an extended Higgs sector. Moreover, peculiar signatures from stop decays could also characterise the light singlino scenario.

\subsection{Region 2, $45<m_{\tilde{\chi}_{1}^{0}}<55 \mathrm{GeV}$}

This region is characterised by a large $m_{0} \sim 10^{5} \mathrm{GeV}$ and a small $M_{1 / 2} \lesssim 500 \mathrm{GeV}$. The LSP is almost an equal admixture of higgsino and singlino, with a bino component $\lesssim 20 \%$. The DM relic density is always below $\Omega h^{2}<0.01$, see figure 1. Due to the high value of $m_{0}$, all sfermions are heavy and decoupled and the only sub-TeV sparticles are EWinos and gluinos. The EWinos are rather mixed states, with a mass $\lesssim 200 \mathrm{GeV}$, while the gluino lies in the $800-1500 \mathrm{GeV}$ mass range. The lightest CP even Higgs state, $h_{1}$, is SM like, and all the other Higgs states are heavier than $300 \mathrm{GeV}$, with $h_{2}$ and $a_{1}$ carrying a dominant singlet component if lighter than $600 \mathrm{GeV}$ (otherwise $h_{3}$ and $a_{2}$ are the dominant singlet states). 

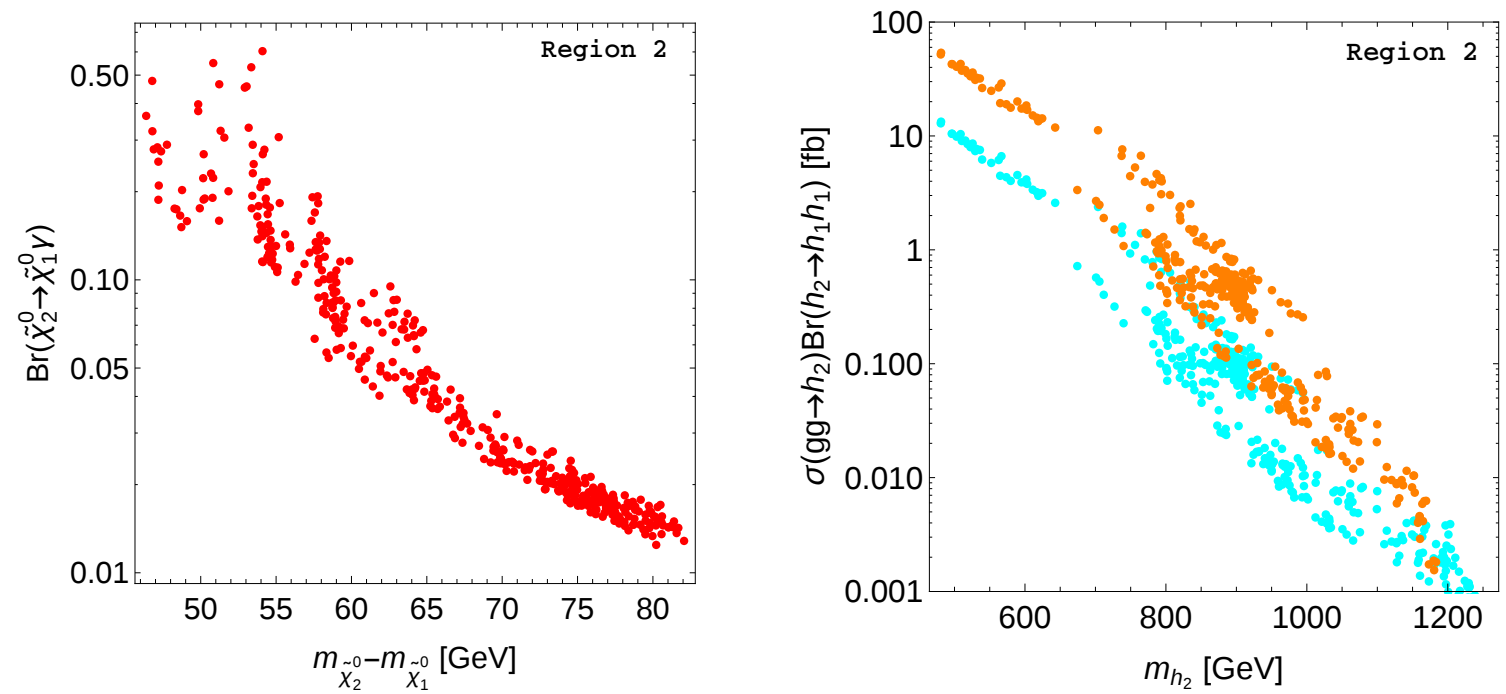

Figure 5. $\operatorname{Br}\left(\tilde{\chi}_{2}^{0} \rightarrow \gamma \tilde{\chi}_{1}^{0}\right)$ in function of $m_{\tilde{\chi}_{2}^{0}}-m_{\tilde{\chi}_{1}^{0}}($ left $)$ and $\sigma\left(g g \rightarrow h_{2}\right) \operatorname{Br}\left(h_{2} \rightarrow h_{1} h_{1}\right)$ in function of $m_{h_{2}}$ (right) for the 8 and $13 \mathrm{GeV}$ LHC (cyan and orange points respectively). Both panels include only points in region 2 for which $m_{\tilde{g}} \gtrsim 1100 \mathrm{GeV}$. The LSP mass is $\sim 45-55 \mathrm{GeV}$.

To check the LHC Run-1 reach on the gluino sector, we have exploited the same technique used in section 4.1.1, based on the recast of the CMS gluino search [99] implemented in MadAnalysis5, and obtained a similar limit of $m_{\tilde{g}} \gtrsim 1100 \mathrm{GeV}$.

Using SModelS we did not find any additional constraint arising from the EWino sector. The most sensitive analysis, among the ones implemented in SModelS, is the CMS search for $\tilde{\chi}_{2}^{0} \tilde{\chi}_{1}^{ \pm}$production in $W^{ \pm} Z+E_{T}^{\text {miss }}$ final state with either on-shell or off-shell SM gauge bosons [115]. However, in this region of the nMSSM parameter space, the masses of the EWinos are approximately $m_{\tilde{\chi}_{1}^{ \pm}} \sim 103-120 \mathrm{GeV}$ and $m_{\tilde{\chi}_{2}^{0}} \sim 80-120 \mathrm{GeV}$. Their decays therefore occur via an off-shell $W$ or $Z$, falling close to the region where the CMS search looses sensitivity, $m_{\tilde{\chi}_{2}^{0}}=m_{\tilde{\chi}_{1}^{0}}+m_{Z}$. Another reason for this region not to be constrained by EWino searches is that there are other important decay channels for the neutralinos, which lead to a reduction of the branching into a $\mathrm{Z}$ boson. Notably, $\tilde{\chi}_{2}^{0}$ can decay into a $\gamma \tilde{\chi}_{1}^{0}$ final state, with a branching ratio up to $50 \%$ for a mass splitting between $\tilde{\chi}_{2}^{0}$ and $\tilde{\chi}_{1}^{0}$ of $\sim 50 \mathrm{GeV}$, as shown in figure 5 (left panel). This opens up the interesting possibility of looking at $\tilde{\chi}_{1}^{ \pm} \chi_{2}^{0}$ production, for which the cross section can be close to $\mathcal{O}(1 \mathrm{pb})$ already at LHC Run-1, in either the $2 j+\gamma+E_{T}^{\text {miss }}$ or $l+\gamma+E_{T}^{\text {miss }}$ final states. Interestingly, the photon might have a sizeable $p_{T}$, as the $\tilde{\chi}_{2}^{0}-\tilde{\chi}_{1}^{0}$ mass difference can be $\mathcal{O}(50 \mathrm{GeV})$. However such a mass splitting does not allow for large $E_{T}^{\text {miss }}$, therefore it might be necessary to trigger on an ISR jet. We leave this point for further investigation and we provide one benchmark point for this scenario in table 4 (BMP2-I).

In this region the extra Higgs states $h_{2}$ and $a_{1}$ are either doublet or singlet like, depending on their masses, and almost degenerate. Only the states heavier than $\sim 450 \mathrm{GeV}$ survive LHC-Run 1 constraints, set by gluino searches. Possible decay patterns that have been searched for at the LHC are $a_{1} \rightarrow Z h_{1}$ and $h_{2} \rightarrow h_{1} h_{1}$ [116-118], which do not 


\begin{tabular}{|c|c|c|}
\hline & BMP2-I & BMP2-II \\
\hline $\tan \beta$ & 1.7 & 1.82 \\
\hline$\lambda$ & 0.73 & 0.72 \\
\hline$\mu$ & 130 & 126.0 \\
\hline$m_{0}$ & $2.34 \cdot 10^{5}$ & $5.3 \cdot 10^{4}$ \\
\hline$M_{1 / 2}$ & 269 & 326 \\
\hline$A_{0}$ & -5879 & -3180 \\
\hline$A_{\lambda}$ & 0 & 0 \\
\hline$\xi_{F}$ & $5.46 \cdot 10^{5}$ & $1.52 \cdot 10^{5}$ \\
\hline$\xi_{S}$ & -301 & -2971 \\
\hline Masses & $m_{\tilde{\chi}_{1}^{ \pm}}=105, m_{\tilde{\chi}_{2}^{0}}=102$ & $m_{h_{2}}=478, m_{\tilde{\chi}_{1}^{ \pm}}=104$ \\
\hline$\sigma[\mathrm{fb}]$ & $\sigma_{\tilde{\chi}_{1}^{ \pm} \tilde{\chi}_{2}^{0}}^{13(8) \mathrm{TeV}}=1729(860)$ & $\sigma_{g g \rightarrow h_{2}}^{13 \mathrm{TeV}}=524$ \\
\hline $\mathrm{Br}$ & $\operatorname{Br}\left(\tilde{\chi}_{2}^{0} \rightarrow \tilde{\chi}_{1}^{0} \gamma\right)=50 \%$ & $\begin{array}{c}\operatorname{Br}\left(h_{2} \rightarrow t \bar{t}\right)=15 \% \\
\operatorname{Br}\left(h_{2} \rightarrow \tilde{\chi}_{1}^{+} \tilde{\chi}_{1}^{-}\right)=29 \% \\
\operatorname{Br}\left(h_{2} \rightarrow \tilde{\chi}_{i \neq 0}^{0} \tilde{\chi}_{j \neq 0}^{0}\right)=29 \% \\
\operatorname{Br}\left(h_{2} \rightarrow \tilde{\chi}_{1}^{0} \tilde{\chi}_{1}^{0}\right)=6 \% \\
\operatorname{Br}\left(\tilde{\chi}_{1}^{ \pm} \rightarrow \tilde{\chi}_{1}^{0} W^{*}\right)=100 \%\end{array}$ \\
\hline
\end{tabular}

Table 4. Benchmark points choices for region 2. Dimensionful parameter are expressed in GeV, except $\xi_{F}$ and $\xi_{S}$ which are in $\mathrm{GeV}^{2}$ and $\mathrm{GeV}^{3}$ respectively and $\sigma$, expressed in fb. We indicate in the table the relevant mass spectrum, cross sections and branching ratios.

however impose any constraint on this region of the parameter space. This is due to the small $\operatorname{Br}\left(a_{1} \rightarrow Z h_{1}\right)<0.5 \%$ and to the fact that the mass of $h_{2}$ lies beyond the reach of the experimental analysis which is around $360 \mathrm{GeV}$.

We show in figure 5 (right panel) the rates $\sigma\left(g g \rightarrow h_{2}\right) \operatorname{Br}\left(h_{2} \rightarrow h_{1} h_{1}\right)$ for the 8 and $13 \mathrm{TeV}$ LHC (cyan and orange respectively), where the gluon fusion production cross sections have been obtained by rescaling the one provided by the LHC Higgs cross section working group [119] with the reduced $g g h_{2}$ coupling provided by NMSSMTools. Recall that the current limit is $\sigma\left(g g \rightarrow h_{2}\right) \operatorname{Br}\left(h_{2} \rightarrow h_{1} h_{1}\right) \lesssim 4 \mathrm{pb}$ for $m_{h_{2}}=360 \mathrm{GeV}$, thus roughly two orders of magnitude above the predicted value.

Given that the mass of the $h_{2}$ is above the $t \bar{t}$ threshold, of particular interest is its decays into a top pair. The cross section times branching ratio can reach $\mathcal{O}(100 \mathrm{fb})$ for the $13 \mathrm{TeV}$ LHC, as illustrated in figure 6 (left panel), and a similar value is predicted for $a_{1}$. This channel however deserves a deeper investigation, due to the well known possibility of dominant interference with the $t \bar{t}$ QCD background, for which dedicated cuts might need to be applied, see e.g. [120]. In the MSSM, an analysis has shown that the LHC could find evidence of a Higgs boson in this channel for masses up to $1 \mathrm{TeV}$ and low values of $\tan \beta$ [121]. The possibility of $h_{2}$ decaying into a pair of EWinos is also interesting, although it weakens the discovery potential in the $t \bar{t}$ mode. We show as an illustrative example the rates for the process $g g \rightarrow h_{2} \rightarrow \tilde{\chi}_{1}^{+} \tilde{\chi}_{1}^{-}$in figure 6 (right panel) for the 8 and 

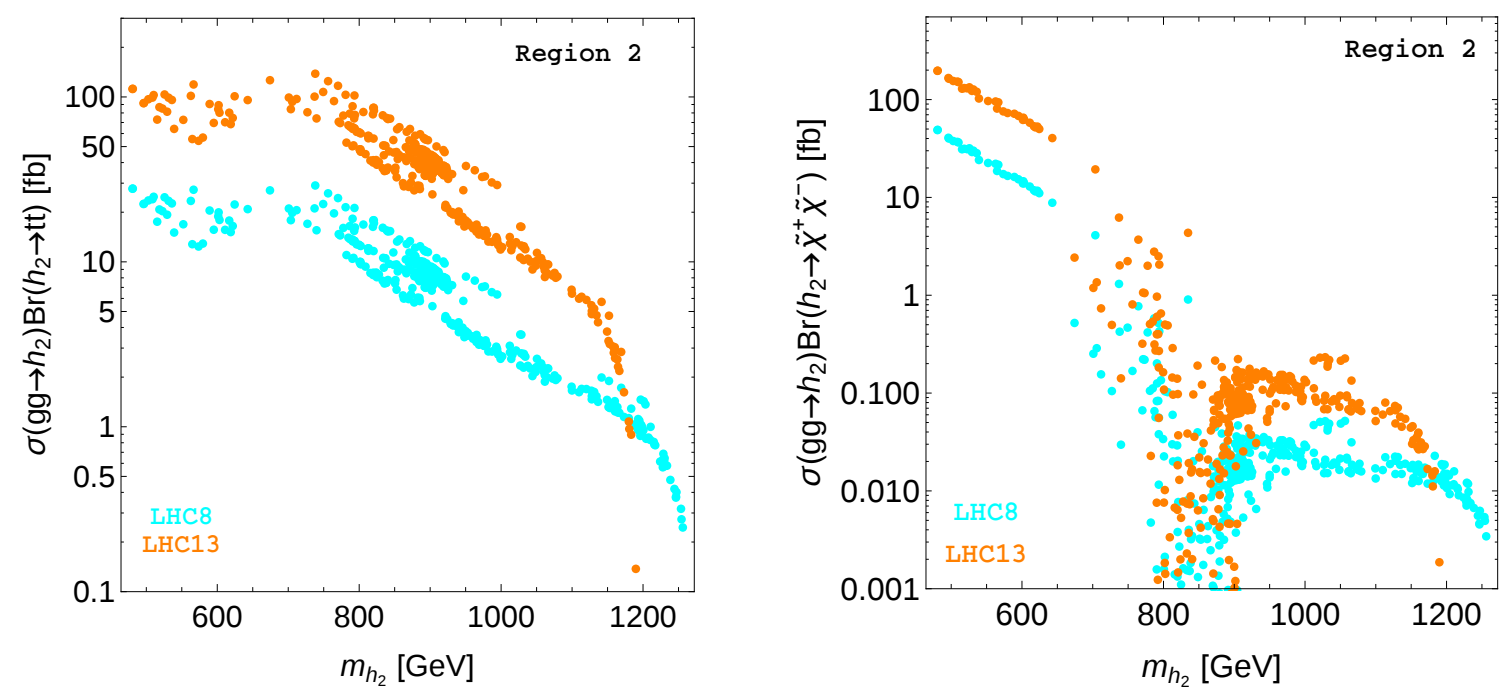

Figure 6. $\sigma\left(g g \rightarrow h_{2}\right) \operatorname{Br}\left(h_{2} \rightarrow t \bar{t}\right)$ (left) and $\sigma\left(g g \rightarrow h_{2}\right) \operatorname{Br}\left(h_{2} \rightarrow \tilde{\chi}_{1}^{+} \tilde{\chi}_{1}^{-}\right)$(right) for the 8 and $13 \mathrm{TeV}$ LHC (cyan and orange points respectively) as function of $m_{h_{2}}$.

$13 \mathrm{TeV}$ LHC. For the latter the cross section times branching can reach $\mathcal{O}(100 \mathrm{fb})$ for a $\sim 500 \mathrm{GeV} h_{2}$, giving the possibility of a different search channel for $h_{2}$ and/or $a_{1}$. Clearly, for heavier Higgs states the rate drops rapidly. We provide in table 4 a benchmark point (BMP2-II) that simultaneously maximises the two aforementioned rates. Note that for this point the branching ratio into neutralinos is similar to the one into charginos, while the pure invisible decay has a $6 \%$ branching ratio. Rates for the lightest pseudoscalar $a_{1}$ are somewhat similar. Note that such large branching ratios in EWinos can also be found in the MSSM [122].

Finally, unlike the first region, ID experiments do not set any additional constraints, since the rescaled rate into $b \bar{b}$ final state (the most relevant for the mass range of interest) is much below the current constraint from Fermi-LAT, as shown in figure 7 (left panel). Prospects for DD measurements are once again quite pessimistic, as illustrated in the right panel of figure 7. The rescaled spin independent cross section for DD is more than one order of magnitude below the current limit set by the LUX experiment, and XENON1T will be able to marginally cover only a small region with $m_{\tilde{\chi}_{1}^{0}}>50 \mathrm{GeV}$. Such bleak DD prospects are typical of models where one relies on a resonance to achieve the correct relic density.

\subsection{Region 3, $m_{\tilde{\chi}_{1}^{0}} \sim 65 \mathrm{GeV}$}

The $\sim 65 \mathrm{GeV}$ LSP can appear in different regions of the $m_{0}-M_{1 / 2}$ parameter space, in which it will have a different composition. We have identified the following three regions:

- Region 3A, large $m_{0}\left(\sim 10^{4} \mathrm{GeV}\right)$ and small $M_{1 / 2}(\lesssim 200 \mathrm{GeV})$. In this region the LSP is mainly bino and the DM relic density can be compatible with the value measured by Planck, see figure 1 . 

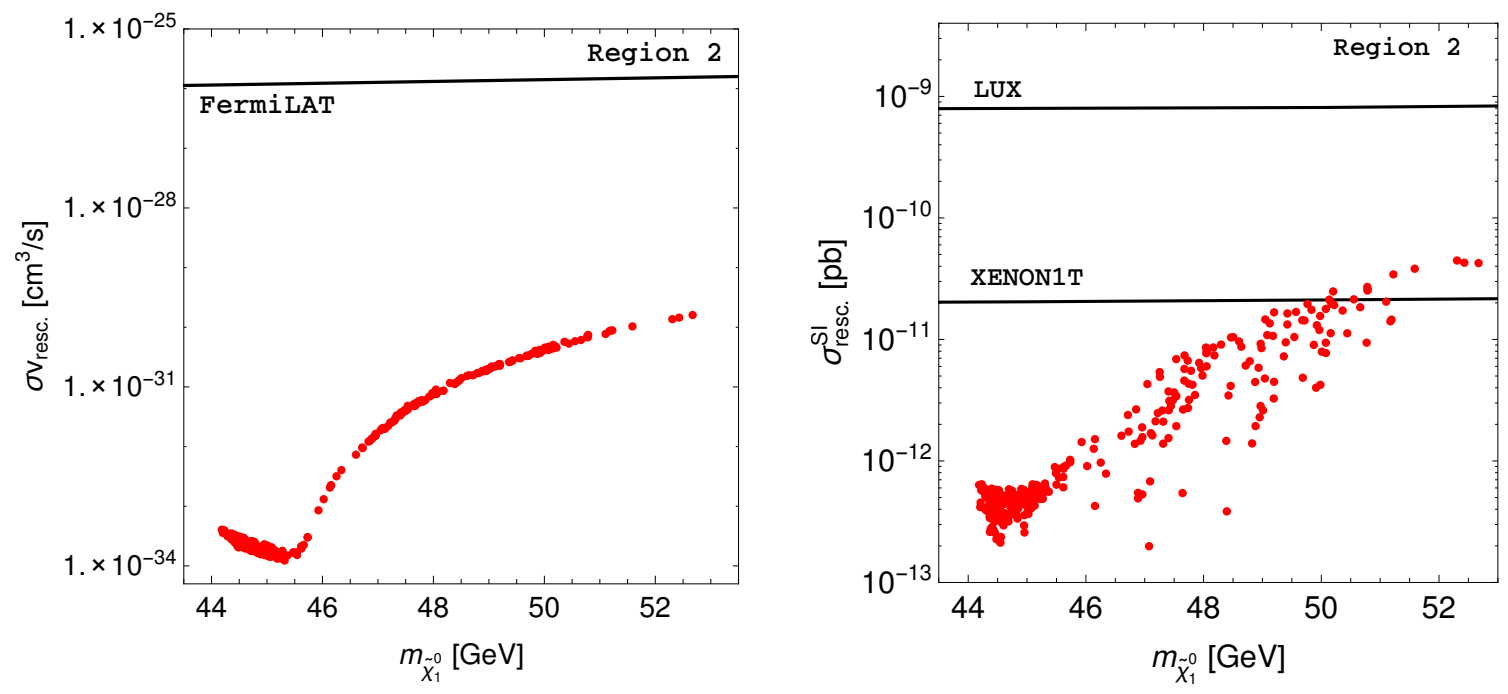

Figure 7. ID detection rate into $b \bar{b}$ final state (left) and rescaled spin independent cross section for DD (right) in function of $m_{\tilde{\chi}_{1}^{0}}$. The horizontal lines represent the current limit from FermiLAT (left) and LUX (right). Also shown in the right plot is the projection for XENON1T experiment. Only the points surviving the LHC Run-1 constraints are shown.

- Region 3B, $m_{0} \sim 1 \mathrm{TeV}$ and $M_{1 / 2} \lesssim 500 \mathrm{GeV}$. In this region the LSP is a mixed higgsino and singlino state.

- Region 3C, small $m_{0}(\lesssim 1 \mathrm{TeV})$ and large $M_{1 / 2}\left(\sim 10^{4} \mathrm{GeV}\right)$. Here the LSP is also a higgsino/singlino admixture.

\subsubsection{Region 3A and 3B: bino or higgsino/singlino LSP with low $M_{1 / 2}$}

In region $3 \mathrm{~A}$ and $3 \mathrm{~B}$ the relic density is kept to a small value by annihilation of the LSP through the $\mathrm{Z}$ boson or the SM like Higgs boson $h_{1}$. The very small higgsino component of the LSP in region $3 \mathrm{~A}$ (around $2 \%$ ) is just sufficient to provide a DM relic density $\Omega h^{2} \approx 0.1$. The heavier CP even state, $h_{2}$, has a mass between 250 and $4000 \mathrm{GeV}$, almost degenerate with $a_{1}$, in both regions. Considering the values of $m_{0}$ and $M_{1 / 2}$, the SUSY spectrum is characterised by light EWinos and light gluinos. Other sfermions are above the TeV scale and decoupled in region 3A while squarks, especially stops, can be below the TeV scale in region $3 \mathrm{~B}$.

In region $3 \mathrm{~A}$ all the EWinos are lighter than $200 \mathrm{GeV}$, and the gluino is always lighter than $600 \mathrm{GeV}$. This region is therefore already completely ruled out by the LHC Run-1 searches for gluinos. Indeed, with the same procedure as in section 4.1.1, we have checked that gluino masses $\lesssim 1100 \mathrm{GeV}$ are excluded. Recall that in a model with gaugino mass unification at the GUT scale, the gluino and bino masses are respectively $m_{\tilde{g}} \approx 3 M_{1 / 2}$ and $m_{\tilde{B}} \approx 1 / 6 M_{1 / 2}$, thus the upper limit on the (bino) LSP mass set by the upper limit on the singlino mass from eq. (2.18), entails an upper limit on the gluino mass. This has the important consequence of leaving just region 1, the one with the very light singlino LSP, as an nMSSM explanation for the entire relic abundance of the universe, together with a heavy enough gluino to pass LHC Run-1 constraints. 
The same bound on the gluino mass has also a strong impact in region 3B. Here the value of $M_{1 / 2}$ is slightly higher than in region $3 \mathrm{~A}$, thus the gluino is somewhat heavier, $600<m_{\tilde{g}}<1200 \mathrm{GeV}$. Nevertheless a large part of this region is already excluded by LHC Run-1. In addition, all EWinos except the heavier neutral and charged states, have a mass below $200 \mathrm{GeV}$, and are slightly constrained by direct EWinos searches, though the main constraint remains the one on the gluino mass. Moreover the lightest stop lies in the 200-500 GeV range because of the smaller value of $m_{0}$. In principle, for a $60 \mathrm{GeV}$ LSP such a light stops could be excluded. However this is not the case here for two reasons. The first is that stops with a mass $\sim 250 \mathrm{GeV}$ are close to the kinematic edge $m_{\tilde{t}}-m_{\tilde{\chi}_{1}^{0}}=m_{Z}$ (see for example refs. $[96,123])$ where standard stop searches loose sensitivity, causing therefore a poor exclusion limit. The second is that for higher values of the stop mass, around $400 \mathrm{GeV}$, the same analyses loose sensitivity since the stop decays do not fulfill the simplified model assumptions. In particular, the branching ratios $\operatorname{Br}\left(\tilde{t} \rightarrow \tilde{\chi}_{1}^{ \pm} b\right)$ and $\operatorname{Br}\left(\tilde{t} \rightarrow \tilde{\chi}_{1}^{0} t\right)$ are both suppressed. The reason is that the coupling of the stop to the singlino is small, hence other decay channels into heavier neutralinos are favoured despite the reduced phase space, causing therefore a reduction of the exclusion power of the experimental analyses. The two previous statements about the non exclusion of such light stops have been checked via the CMS search [96] available in the MadAnalysis5 Public Analysis Database [124]. The reinterpretation of the LHC Run-1 results on gluino searches leave therefore just a small window of parameter space available, namely for $m_{\tilde{g}} \gtrsim 1100 \mathrm{GeV}$.

While extra Higgs searches such as $a_{1} \rightarrow Z h_{1}$ and $h_{2} \rightarrow h_{1} h_{1}$ do not set any constraint on this region of parameter space, ${ }^{5}$ exotic decays of heavy Higgs states in a pair of EWinos can have substantial rates. This leads again to the interesting possibility of searching for these states through their EWino decay channels. Cross sections of $\mathcal{O}(100 \mathrm{fb})$ are expected for $g g \rightarrow h_{2}, h_{2} \rightarrow \tilde{\chi}_{1}^{+} \tilde{\chi}_{1}^{-}$at the $13 \mathrm{TeV}$ LHC, with a $h_{2} \sim 300 \mathrm{GeV}$. We provide one such benchmark in table 5 (BMP3-I). Note that despite of a $h_{2}$ mass below $300 \mathrm{GeV}$, this point is safely below the recent limits of CMS in the $W W$ channel [83].

As concerns ID measurements, the relevant rates are well below the limits set by the Fermi-LAT experiment. Prospects for future underground experiments for DD are quite interesting. The SI cross section lies just below the limits set by the LUX experiment and is roughly constant in this region. It is determined essentially by the $h_{1}$ coupling to the LSP which does not vary much in this region. The rescaled cross section is however suppressed by up to almost two orders of magnitude, since the thermally averaged DM annihilation cross section is strongly enhanced when $m_{h_{1}}-2 m_{\tilde{\chi}_{1}^{0}}$ is $\sim 1 \mathrm{GeV}$, see figure 8 . Nevertheless, as shown in this figure, XENON1T will be able to probe all this region of parameter space, offering a valid complement to collider based analyses.

\subsubsection{Region 3C: higgsino/singlino LSP with high $M_{1 / 2}$}

This region at high $M_{1 / 2}$ and low $m_{0}$ is characterised by light sleptons and light higgsinos and singlino, while the bino and wino states are heavy and decoupled. Squarks and gluinos

\footnotetext{
${ }^{5}$ Note that the main search channel at low values of $\tan \beta$ is in gauge bosons and that the constraints on the SM like Higgs imply a suppressed coupling of the heavy Higgs to gauge bosons, as in the MSSM. The suppression is even stronger for the singlet Higgs.
} 


\begin{tabular}{|l|c|}
\hline & BMP3B-I \\
\hline $\tan \beta$ & 1.96 \\
\hline$\lambda$ & 0.688 \\
\hline$\mu$ & -105 \\
\hline$m_{0}$ & 260 \\
\hline$M_{1 / 2}$ & 529 \\
\hline$A_{0}$ & 3042 \\
\hline$A_{\lambda}$ & 1489 \\
\hline$\xi_{F}$ & $1.66 \cdot 10^{6}$ \\
\hline$\xi_{S}$ & $3.82 \cdot 10^{8}$ \\
\hline \hline Masses & $m_{h_{2}}=276, m_{\tilde{\chi}_{1}^{ \pm}}=115$ \\
\hline$\sigma[\mathrm{fb}]$ & $\sigma_{g g \rightarrow h_{2}}^{13 \mathrm{TeV}}=180$ \\
\hline $\operatorname{Br}$ & $\operatorname{Br}\left(h_{2} \rightarrow \tilde{\chi}_{1}^{+} \tilde{\chi}_{1}^{-}\right)=51 \%$ \\
& $\operatorname{Br}\left(\tilde{\chi}_{1}^{ \pm} \rightarrow \tilde{\chi}_{1}^{0} W^{*}\right)=100 \%$ \\
\hline
\end{tabular}

Table 5. Benchmark points choices for region 3B. Dimensionful parameter are expressed in $\mathrm{GeV}$, except $\xi_{F}$ and $\xi_{S}$ which are in $\mathrm{GeV}^{2}$ and $\mathrm{GeV}^{3}$ respectively and $\sigma$, expressed in fb. We indicate in the table the relevant mass spectrum, cross sections and branching ratios.

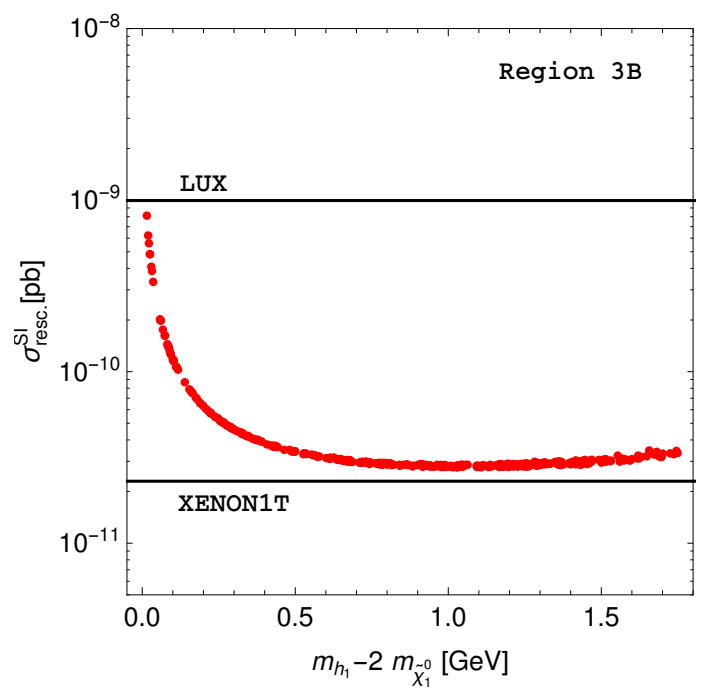

Figure 8. Spin independent cross section for DD as function of $m_{h_{1}}-2 m_{\tilde{\chi}_{1}^{0}}$, for all the points $m_{\tilde{\chi}_{1}^{0}} \approx 60 \mathrm{GeV}$ The horizontal lines represent the current limit from LUX and the projected limits for the XENON1T experiment.

are also heavy and decoupled, and the Higgs states $h_{2}$ and $a_{1}$ have a mass greater than $600 \mathrm{GeV}$. Note that even though $m_{0}$ is small at the GUT scale, the squarks are heavy because the renormalisation group equations that are used to derive their mass at the SUSY scale receive important contributions from $M_{1 / 2}$ which is large. 


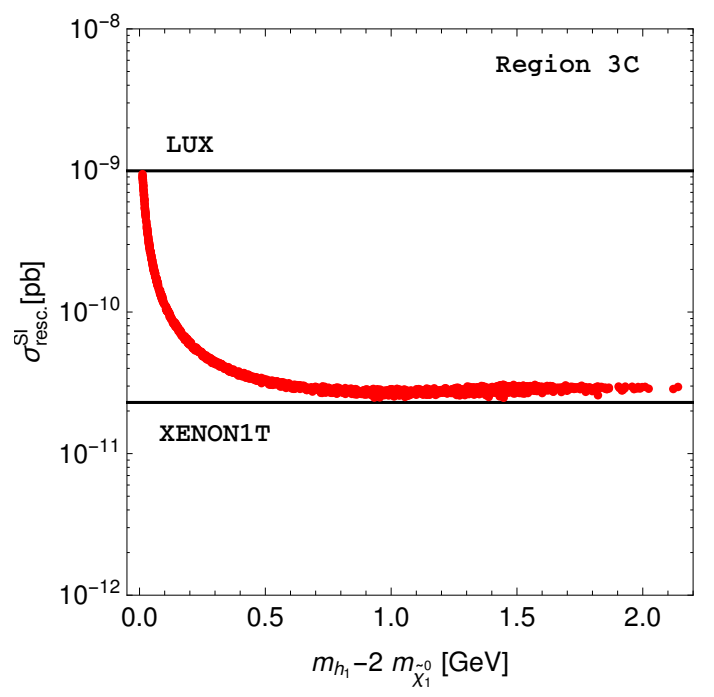

Figure 9. Spin independent cross section for DD as function of $m_{h_{1}}-2 m_{\tilde{\chi}_{1}^{0}}$. The horizontal lines represent the current limit from LUX and the projected limits for the XENON1T experiment.

Despite the light EWinos, $m_{\tilde{\chi}_{2}^{0}, \tilde{\chi}_{1}^{ \pm}} \sim 150 \mathrm{GeV}$, sleptons, $m_{e_{R} / \mu_{R}} \sim 200 \mathrm{GeV}$, and $\tilde{\tau}_{1}$ as light $100 \mathrm{GeV}$, no constraints are obtained by the LHC Run-1 SUSY searches. First and second generation right handed charged sleptons with a mass $100-300 \mathrm{GeV}$ for a $\sim 65 \mathrm{GeV}$ LSP lie on the edge of the exclusion set by the ATLAS slepton search with $2 l+E_{T}^{\text {miss }}$ final state [95]. The limit set by ATLAS on the $\tilde{\tau}^{+} \tilde{\tau}^{-}$production cross section varies between $0.76 \mathrm{pb}$ for $m_{\tilde{\tau}}=100 \mathrm{GeV}$ and $0.02 \mathrm{pb}$ for $m_{\tilde{\tau}}=300 \mathrm{GeV}$ for a $60 \mathrm{GeV}$ LSP [125]. This limit is roughly one order of magnitude larger than the predicted values, therefore making this search not sensitive to this scenario.

Since the EWinos are mostly higgsino or singlino, their production cross sections are small. Moreover, the $\tilde{\chi}_{1,2}^{0}$ and $\tilde{\chi}_{1}^{+}$masses lie close to the kinematic edge for off-shell decay where standard searches loose sensitivity, making it therefore easy to escape the LHC limits. We expect however that upgrade of the standard simplified model searches will be able to cover this region of parameter space with early LHC Run-2 data given the standard decay modes of these particles, and the fact that in this region SModels gives a ratio between the theoretical prediction and the experimental measurement which reaches the value of 0.7 , i.e. not too far from the value of 1 , for which we claim the point to be excluded. Finally, as above, ton-scale underground experiments for DD, such as XENON1T, will be able to fully cover this region of parameter space, see figure 9 , while no constraints are actually enforced by ID measurements.

\section{Conclusions}

In this paper we have explored the parameter space of the nMSSM with input parameters defined at the GUT scale, and identified three broad regions for the LSP mass which satisfy theoretical, astrophysical, cosmological and collider constraints. In particular we have considered collider constraints on the Higgs boson and sparticles, as well as flavour 
constraints available in NMSSMTools, upper limit on DM DD from LUX and relic abundance of DM as measured by Planck. The nMSSM is characterised by the presence of a singlino with a mass below $75 \mathrm{GeV}$, since only mixing with higgsinos contribute to its, otherwise vanishing, mass. In only two of the three regions the lightest neutralino LSP can account completely for the DM abundance. The first features a light singlino LSP $\left(m_{\tilde{\chi}_{1}^{0}} \sim 5 \mathrm{GeV}\right)$ the second a heavier one $\left(m_{\tilde{\chi}_{1}^{0}} \sim 65 \mathrm{GeV}\right)$, mostly bino. In all the other allowed regions the computed relic density lies below the value measured by Planck.

A closer scrutiny at the constraints arising from SUSY searches at the LHC, implemented via two public tools for analyses recast, SModelS and MadAnalysis5, showed that some of these regions (or sub-regions) were actually completely exclude by results of LHC Run-1, notably when they featured a gluino below the $\mathrm{TeV}$ scale. In particular the only valid region not requiring an additional DM component is the one with a light singlino.

We have investigated how the model could be probed at the LHC Run-2 or through non collider DM searches. In particular, the region with a light singlino is characterised by the presence of a light pseudoscalar with a mass which is twice the one of the singlino, and a scalar Higgs with a mass below that of the recently discovered scalar. Hence, searches for these extra light states could lead to a distinctive signal of the nMSSM. Relevant signatures include the decay of the $125 \mathrm{GeV}$ Higgs into light scalars or pseudoscalars, leading for example to $4 b, 2 b / 2 \tau$ or $4 \tau$ final states, or the direct production of one of the light scalar or pseudoscalar. Some of these signatures are under investigation at LHC, since the possibility of a light CP odd Higgs is one of the hallmark signature of generic NMSSM, and searches for light Higgs bosons are a priority in the LHC Run-2 program.

In other regions where the lightest Higgs is the already discovered boson at $125 \mathrm{GeV}$, the model can be probed via searches for the heavy Higgs states. One characteristic of the model, as opposed to the MSSM but shared with the generic NMSSM, is that values of $\tan \beta \approx 1-2$ can be compatible with all the present constraints. Thus, searches for heavy Higgs states decaying into top pairs will provide further probes of the model. Another characteristic feature is the possibility of a heavy Higgs state decaying with a high rate into a pair of charginos or neutralinos. In particular we have found large rates even with a Higgs mass below $300 \mathrm{GeV}$. While a large branching ratio into charginos for the heavy Higgs state can occur in the MSSM, the Higgs mass is typically much higher [122], hence this process suffers from suppression due to the somewhat smaller cross section.

In the nMSSM the decays of sparticles have also peculiar features, thus searches for SUSY in Run-2 of the LHC could provide not only a validation of the theoretical SUSY framework, but also a sign of its non-minimality. The most distinctive feature occurs once again in the case of a light singlino LSP. Since the singlino is very weakly coupled to the sfermions, the decay channels involving the singlino in the final state typically have small partial widths. Therefore decays of sfermions, and in particular squarks, proceed preferably through the heavy neutralinos rather than directly in the LSP. Moreover the heavier neutralinos and charginos can decay into the LSP and a gauge boson (always permitted by phase space) and into a LSP and a light scalar or pseudoscalar. When the singlino LSP is not so light, we have found another unusual signature corresponding to $\tilde{\chi}_{2}^{0}$, which can decay with a substantial rate into a $\tilde{\chi}_{1}^{0} \gamma$ final state even when there is a considerable mass 
difference between these two states. Of course conventional SUSY searches at LHC Run-2 will probe further the allowed parameter space of the model, notably through searches for squarks and gluinos but also EWinos and sleptons.

We have also pointed out that DD searches are complementary to collider searches, and that in particular a ton-scale detector could completely probe the remaining allowed region with a LSP around $65 \mathrm{GeV}$, while large signals in ID are not expected, except in very specific kinematic configurations. A thorough investigation of these new signatures at the LHC, which lies beyond the scope of this work, is required to assess the potential of the LHC to discover and/or probe the nMSSM.

\section{Acknowledgments}

DB thanks Ursula Laa for useful discussions regarding the use of SModelS. This work was supported in part by the LIA-TCAP of CNRS, by the French ANR, Project DMAstroLHC, ANR-12-BS05-0006, by the Investissements d'avenir, Labex ENIGMASS. The work of AP was also supported by the Russian foundation for Basic Research, grant RFBR-1552-16021-CNRS-a. The authors acknowledge the support of France Grilles for providing cloud computing resources on the French National Grid Infrastructure.

Open Access. This article is distributed under the terms of the Creative Commons Attribution License (CC-BY 4.0), which permits any use, distribution and reproduction in any medium, provided the original author(s) and source are credited.

\section{References}

[1] ATLAS collaboration, Observation of a new particle in the search for the standard model Higgs boson with the ATLAS detector at the LHC, Phys. Lett. B 716 (2012) 1 [arXiv: 1207.7214] [INSPIRE].

[2] CMS collaboration, Observation of a new boson at a mass of $125 \mathrm{GeV}$ with the CMS experiment at the LHC, Phys. Lett. B 716 (2012) 30 [arXiv:1207.7235] [INSPIRE].

[3] R. Barbieri and G.F. Giudice, Upper bounds on supersymmetric particle masses, Nucl. Phys. B 306 (1988) 63 [InSPIRE].

[4] L.J. Hall, D. Pinner and J.T. Ruderman, A natural SUSY Higgs near 126 GeV, JHEP 04 (2012) 131 [arXiv:1112.2703] [INSPIRE].

[5] M. Maniatis, The next-to-minimal supersymmetric extension of the standard model reviewed, Int. J. Mod. Phys. A 25 (2010) 3505 [arXiv:0906.0777] [InSPIRE].

[6] U. Ellwanger, C. Hugonie and A.M. Teixeira, The next-to-minimal supersymmetric standard model, Phys. Rept. 496 (2010) 1 [arXiv:0910.1785] [InSPIRE].

[7] U. Ellwanger, G. Espitalier-Noel and C. Hugonie, Naturalness and fine tuning in the NMSSM: implications of early LHC results, JHEP 09 (2011) 105 [arXiv:1107.2472] [INSPIRE].

[8] J.-J. Cao, Z.-X. Heng, J.M. Yang, Y.-M. Zhang and J.-Y. Zhu, A SM-like Higgs near $125 \mathrm{GeV}$ in low energy SUSY: a comparative study for MSSM and NMSSM, JHEP 03 (2012) 086 [arXiv:1202.5821] [INSPIRE]. 
[9] U. Ellwanger and C. Hugonie, Higgs bosons near $125 \mathrm{GeV}$ in the NMSSM with constraints at the GUT scale, Adv. High Energy Phys. 2012 (2012) 625389 [arXiv:1203.5048] [INSPIRE].

[10] M. Perelstein and B. Shakya, XENON100 implications for naturalness in the MSSM, NMSSM and $\lambda$-supersymmetry model, Phys. Rev. D 88 (2013) 075003 [arXiv:1208.0833] [INSPIRE].

[11] K. Agashe, Y. Cui and R. Franceschini, Natural islands for a $125 \mathrm{GeV}$ Higgs in the scale-invariant NMSSM, JHEP 02 (2013) 031 [arXiv:1209.2115] [INSPIRE].

[12] T. Gherghetta, B. von Harling, A.D. Medina and M.A. Schmidt, The scale-invariant NMSSM and the $126 \mathrm{GeV}$ Higgs boson, JHEP 02 (2013) 032 [arXiv:1212.5243] [INSPIRE].

[13] T. Cheng, J. Li, T. Li and Q.-S. Yan, Natural NMSSM confronting with the LHC7-8, Phys. Rev. D 89 (2014) 015015 [arXiv: 1304.3182] [INSPIRE].

[14] D. Kim, P. Athron, C. Balázs, B. Farmer and E. Hutchison, Bayesian naturalness of the CMSSM and CNMSSM, Phys. Rev. D 90 (2014) 055008 [arXiv:1312.4150] [INSPIRE].

[15] A. Fowlie, Is the CNMSSM more credible than the CMSSM?, Eur. Phys. J. C 74 (2014) 3105 [arXiv:1407.7534] [INSPIRE].

[16] J.E. Kim and H.P. Nilles, The $\mu$-problem and the strong CP-problem, Phys. Lett. B 138 (1984) 150 [INSPIRE].

[17] S. Munir, L. Roszkowski and S. Trojanowski, Simultaneous enhancement in $\gamma \gamma, b \bar{b}$ and $\tau^{+} \tau^{-}$rates in the NMSSM with nearly degenerate scalar and pseudoscalar Higgs bosons, Phys. Rev. D 88 (2013) 055017 [arXiv: 1305.0591] [InSPIRE].

[18] G. Bélanger, V. Bizouard and G. Chalons, Boosting Higgs boson decays into gamma and a $Z$ in the NMSSM, Phys. Rev. D 89 (2014) 095023 [arXiv: 1402.3522] [InSPIRE].

[19] U. Ellwanger and A.M. Teixeira, NMSSM with a singlino LSP: possible challenges for searches for supersymmetry at the LHC, JHEP 10 (2014) 113 [arXiv:1406.7221] [INSPIRE].

[20] K.S. Jeong, Y. Shoji and M. Yamaguchi, Higgs mixing in the NMSSM and light Higgsinos, JHEP 11 (2014) 148 [arXiv:1407.0955] [InSPIRE].

[21] S.F. King, M. Mühlleitner, R. Nevzorov and K. Walz, Discovery prospects for NMSSM Higgs bosons at the high-energy Large Hadron Collider, Phys. Rev. D 90 (2014) 095014 [arXiv:1408.1120] [INSPIRE].

[22] U. Ellwanger and A.M. Teixeira, Excessive Higgs pair production with little MET from squarks and gluinos in the NMSSM, JHEP 04 (2015) 172 [arXiv:1412.6394] [INSPIRE].

[23] N.-E. Bomark, S. Moretti and L. Roszkowski, Detection prospects of light NMSSM Higgs pseudoscalar via cascades of heavier scalars from vector boson fusion and Higgs-strahlung, arXiv: 1503.04228 [INSPIRE].

[24] A. Chakraborty, D.K. Ghosh, S. Mondal, S. Poddar and D. Sengupta, Probing the NMSSM via Higgs boson signatures from stop cascade decays at the LHC, Phys. Rev. D 91 (2015) 115018 [arXiv:1503.07592] [InSPIRE].

[25] C.T. Potter, Natural NMSSM with a light singlet Higgs and singlino LSP, arXiv: 1505.05554 [INSPIRE].

[26] D. Das, U. Ellwanger and A.M. Teixeira, Modified signals for supersymmetry in the NMSSM with a singlino-like LSP, JHEP 04 (2012) 067 [arXiv:1202 .5244] [inSPIRE]. 
[27] G. Bélanger, F. Boudjema, C. Hugonie, A. Pukhov and A. Semenov, Relic density of dark matter in the NMSSM, JCAP 09 (2005) 001 [hep-ph/0505142] [INSPIRE].

[28] D.A. Vasquez et al., The $125 \mathrm{GeV}$ Higgs in the NMSSM in light of LHC results and astrophysics constraints, Phys. Rev. D 86 (2012) 035023 [arXiv:1203.3446] [INSPIRE].

[29] C. Hugonie, G. Bélanger and A. Pukhov, Dark matter in the constrained NMSSM, JCAP 11 (2007) 009 [arXiv: 0707.0628] [InSPIRE].

[30] G. Bélanger, C. Hugonie and A. Pukhov, Precision measurements, dark matter direct detection and LHC Higgs searches in a constrained NMSSM, JCAP 01 (2009) 023 [arXiv: 0811.3224] [INSPIRE].

[31] U. Ellwanger and C. Hugonie, The semi-constrained NMSSM satisfying bounds from the LHC, LUX and Planck, JHEP 08 (2014) 046 [arXiv: 1405.6647] [INSPIRE].

[32] D.A. Vasquez, G. Bélanger, C. Boehm, A. Pukhov and J. Silk, Can neutralinos in the MSSM and NMSSM scenarios still be light?, Phys. Rev. D 82 (2010) 115027 [arXiv: 1009.4380] [INSPIRE].

[33] D. Albornoz Vasquez, G. Bélanger and C. Boehm, Astrophysical limits on light NMSSM neutralinos, Phys. Rev. D 84 (2011) 095008 [arXiv:1107.1614] [INSPIRE].

[34] D. Albornoz Vasquez, G. Bélanger, J. Billard and F. Mayet, Probing neutralino dark matter in the MSSM and the NMSSM with directional detection, Phys. Rev. D 85 (2012) 055023 [arXiv: 1201.6150] [INSPIRE].

[35] C. Panagiotakopoulos and K. Tamvakis, New minimal extension of MSSM, Phys. Lett. B 469 (1999) 145 [hep-ph/9908351] [INSPIRE].

[36] C. Panagiotakopoulos and A. Pilaftsis, Higgs scalars in the minimal nonminimal supersymmetric standard model, Phys. Rev. D 63 (2001) 055003 [hep-ph/0008268] [INSPIRE].

[37] A. Dedes, C. Hugonie, S. Moretti and K. Tamvakis, Phenomenology of a new minimal supersymmetric extension of the standard model, Phys. Rev. D 63 (2001) 055009 [hep-ph/0009125] [INSPIRE].

[38] C. Panagiotakopoulos and A. Pilaftsis, Light charged Higgs boson and supersymmetry, Phys. Lett. B 505 (2001) 184 [hep-ph/0101266] [INSPIRE].

[39] A. Menon, D.E. Morrissey and C.E.M. Wagner, Electroweak baryogenesis and dark matter in the NMSSM, Phys. Rev. D 70 (2004) 035005 [hep-ph/0404184] [InSPIRE].

[40] V. Barger, P. Langacker and H.-S. Lee, Lightest neutralino in extensions of the MSSM, Phys. Lett. B 630 (2005) 85 [hep-ph/0508027] [INSPIRE].

[41] V. Barger, P. Langacker, H.-S. Lee and G. Shaughnessy, Higgs sector in extensions of the MSSM, Phys. Rev. D 73 (2006) 115010 [hep-ph/0603247] [InSPIRE].

[42] S.J. Huber, T. Konstandin, T. Prokopec and M.G. Schmidt, Electroweak phase transition and baryogenesis in the NMSSM, Nucl. Phys. B 757 (2006) 172 [hep-ph/0606298] [INSPIRE].

[43] V. Barger, P. Langacker and G. Shaughnessy, Neutralino signatures of the singlet extended MSSM, Phys. Lett. B 644 (2007) 361 [hep-ph/0609068] [INSPIRE].

[44] V. Barger, P. Langacker and G. Shaughnessy, Collider signatures of singlet extended Higgs sectors, Phys. Rev. D 75 (2007) 055013 [hep-ph/0611239] [INSPIRE]. 
[45] V. Barger et al., Recoil detection of the lightest neutralino in MSSM singlet extensions, Phys. Rev. D 75 (2007) 115002 [hep-ph/0702036] [INSPIRE].

[46] C. Balázs, M. Carena, A. Freitas and C.E.M. Wagner, Phenomenology of the NMSSM from colliders to cosmology, JHEP 06 (2007) 066 [arXiv:0705.0431] [INSPIRE].

[47] S.W. Ham, J.O. Im and S.K. Oh, Electroweak phase transition in the MNMSSM with explicit CP-violation, arXiv:0707.4543 [INSPIRE].

[48] S.J. Huber and T. Konstandin, Production of gravitational waves in the NMSSM, JCAP 05 (2008) 017 [arXiv: 0709.2091] [InSPIRE].

[49] E.J. Chun and P. Roy, Dirac leptogenesis in extended NMSSM, JHEP 06 (2008) 089 [arXiv:0803.1720] [INSPIRE].

[50] S.W. Ham, J.O. Im and S.K. Oh, Neutral Higgs bosons in the MNMSSM with explicit CP-violation, Eur. Phys. J. C 58 (2008) 579 [arXiv:0805.1115] [InSPIRE].

[51] J. Cao, H.E. Logan and J.M. Yang, Experimental constraints on NMSSM and implications on its phenomenology, Phys. Rev. D 79 (2009) 091701 [arXiv:0901.1437] [INSPIRE].

[52] J. Cao, K.-i. Hikasa, W. Wang, J.M. Yang and L.-X. Yu, SUSY dark matter in light of CDMS II results: a comparative study for different models, JHEP 07 (2010) 044 [arXiv: 1005.0761] [INSPIRE].

[53] K. Ishikawa, T. Kitahara and M. Takimoto, Singlino resonant dark matter and $125 \mathrm{GeV}$ Higgs boson in high-scale supersymmetry, Phys. Rev. Lett. 113 (2014) 131801 [arXiv:1405.7371] [INSPIRE].

[54] S. Hesselbach, D.J. Miller, G. Moortgat-Pick, R. Nevzorov and M. Trusov, Theoretical upper bound on the mass of the LSP in the MNSSM, Phys. Lett. B 662 (2008) 199 [arXiv:0712.2001] [INSPIRE].

[55] U. Ellwanger and C. Hugonie, NMHDECAY 2.0: an updated program for sparticle masses, Higgs masses, couplings and decay widths in the NMSSM,

Comput. Phys. Commun. 175 (2006) 290 [hep-ph/0508022] [INSPIRE].

[56] U. Ellwanger and C. Hugonie, NMSPEC: a Fortran code for the sparticle and Higgs masses in the NMSSM with GUT scale boundary conditions, Comput. Phys. Commun. 177 (2007) 399 [hep-ph/0612134] [INSPIRE].

[57] S. Kraml et al., SModelS v1.0: a short user guide, arXiv:1412.1745 [INSPIRE].

[58] S. Kraml et al., SModelS: a tool for interpreting simplified-model results from the LHC and its application to supersymmetry, Eur. Phys. J. C 74 (2014) 2868 [arXiv:1312.4175] [INSPIRE].

[59] E. Conte, B. Fuks and G. Serret, MadAnalysis 5, a user-friendly framework for collider phenomenology, Comput. Phys. Commun. 184 (2013) 222 [arXiv:1206.1599] [INSPIRE].

[60] E. Conte, B. Dumont, B. Fuks and C. Wymant, Designing and recasting LHC analyses with MadAnalysis 5, Eur. Phys. J. C 74 (2014) 3103 [arXiv:1405.3982] [INSPIRE].

[61] B. Dumont et al., Toward a public analysis database for LHC new physics searches using MadAnalysis 5, Eur. Phys. J. C 75 (2015) 56 [arXiv: 1407.3278] [inSPIRE].

[62] G. Bélanger, F. Boudjema, A. Pukhov and A. Semenov, MicrOMEGAs 2.0: a program to calculate the relic density of dark matter in a generic model, Comput. Phys. Commun. 176 (2007) 367 [hep-ph/0607059] [INSPIRE]. 
[63] G. Bélanger, F. Boudjema, A. Pukhov and A. Semenov, Dark matter direct detection rate in a generic model with MicrOMEGAs 2.2, Comput. Phys. Commun. 180 (2009) 747 [arXiv:0803.2360] [INSPIRE].

[64] G. Bélanger, F. Boudjema, A. Pukhov and A. Semenov, MicrOMEGAs4.1: two dark matter candidates, Comput. Phys. Commun. 192 (2015) 322 [arXiv:1407.6129] [InSPIRE].

[65] G. Chalons and A. Semenov, Loop-induced photon spectral lines from neutralino annihilation in the NMSSM, JHEP 12 (2011) 055 [arXiv:1110.2064] [INSPIRE].

[66] D. Das, U. Ellwanger and P. Mitropoulos, A 130 GeV photon line from dark matter annihilation in the NMSSM, JCAP 08 (2012) 003 [arXiv:1206.2639] [INSPIRE].

[67] G. Chalons, M.J. Dolan and C. McCabe, Neutralino dark matter and the Fermi gamma-ray lines, JCAP 02 (2013) 016 [arXiv: 1211.5154] [INSPIRE].

[68] G.F. Giudice and A. Masiero, A natural solution to the $\mu$-problem in supergravity theories, Phys. Lett. B 206 (1988) 480 [InSPIRE].

[69] A. Vilenkin, Cosmic strings and domain walls, Phys. Rept. 121 (1985) 263 [INSPIRE].

[70] S.A. Abel, S. Sarkar and P.L. White, On the cosmological domain wall problem for the minimally extended supersymmetric standard model, Nucl. Phys. B 454 (1995) 663 [hep-ph/9506359] [INSPIRE].

[71] S.A. Abel and P.L. White, Baryogenesis from domain walls in the next-to-minimal supersymmetric standard model, Phys. Rev. D 52 (1995) 4371 [hep-ph/9505241] [INSPIRE].

[72] H.P. Nilles, M. Srednicki and D. Wyler, Constraints on the stability of mass hierarchies in supergravity, Phys. Lett. B 124 (1983) 337 [INSPIRE].

[73] A.B. Lahanas, Light singlet, gauge hierarchy and supergravity, Phys. Lett. B 124 (1983) 341 [INSPIRE].

[74] U. Ellwanger, Nonrenormalizable interactions from supergravity, quantum corrections and effective low-energy theories, Phys. Lett. B 133 (1983) 187 [INSPIRE].

[75] H.P. Nilles and N. Polonsky, Gravitational divergences as a mediator of supersymmetry breaking, Phys. Lett. B 412 (1997) 69 [hep-ph/9707249] [INSPIRE].

[76] J. Bagger and E. Poppitz, Destabilizing divergences in supergravity coupled supersymmetric theories, Phys. Rev. Lett. 71 (1993) 2380 [hep-ph/9307317] [INSPIRE].

[77] V. Jain, On destabilizing divergencies in supergravity models, Phys. Lett. B 351 (1995) 481 [hep-ph/9407382] [INSPIRE].

[78] J. Bagger, E. Poppitz and L. Randall, Destabilizing divergences in supergravity theories at two loops, Nucl. Phys. B 455 (1995) 59 [hep-ph/9505244] [InSPIRE].

[79] C. Panagiotakopoulos and K. Tamvakis, Stabilized NMSSM without domain walls, Phys. Lett. B 446 (1999) 224 [hep-ph/9809475] [INSPIRE].

[80] G. Degrassi and P. Slavich, On the radiative corrections to the neutral Higgs boson masses in the NMSSM, Nucl. Phys. B 825 (2010) 119 [arXiv:0907.4682] [InSPIRE].

[81] F. Staub et al., Higgs mass predictions of public NMSSM spectrum generators, arXiv: 1507.05093 [INSPIRE].

[82] G. Bélanger, B. Dumont, U. Ellwanger, J.F. Gunion and S. Kraml, Global fit to Higgs signal strengths and couplings and implications for extended Higgs sectors, Phys. Rev. D 88 (2013) 075008 [arXiv:1306.2941] [INSPIRE]. 
[83] ATLAS collaboration, Search for a high-mass Higgs boson decaying to a $W$ boson pair in pp collisions at $\sqrt{s}=8 \mathrm{TeV}$ with the ATLAS detector, arXiv:1509.00389 [INSPIRE].

[84] Planck collaboration, R. Adam et al., Planck 2015 results. I. Overview of products and scientific results, arXiv:1502.01582 [INSPIRE].

[85] N. Baro, F. Boudjema and A. Semenov, Full one-loop corrections to the relic density in the MSSM: a few examples, Phys. Lett. B 660 (2008) 550 [arXiv:0710.1821] [INSPIRE].

[86] LUX collaboration, D.S. Akerib et al., First results from the LUX dark matter experiment at the Sanford Underground Research Facility, Phys. Rev. Lett. 112 (2014) 091303 [arXiv: 1310.8214] [INSPIRE].

[87] B.C. Allanach et al., SUSY Les Houches Accord 2, Comput. Phys. Commun. 180 (2009) 8 [arXiv:0801.0045] [INSPIRE].

[88] W. Beenakker, R. Hopker, M. Spira and P.M. Zerwas, Squark and gluino production at hadron colliders, Nucl. Phys. B 492 (1997) 51 [hep-ph/9610490] [InSPIRE].

[89] W. Beenakker, M. Krämer, T. Plehn, M. Spira and P.M. Zerwas, Stop production at hadron colliders, Nucl. Phys. B 515 (1998) 3 [hep-ph/9710451] [INSPIRE].

[90] A. Kulesza and L. Motyka, Threshold resummation for squark-antisquark and gluino-pair production at the LHC, Phys. Rev. Lett. 102 (2009) 111802 [arXiv:0807.2405] [INSPIRE].

[91] A. Kulesza and L. Motyka, Soft gluon resummation for the production of gluino-gluino and squark-antisquark pairs at the LHC, Phys. Rev. D 80 (2009) 095004 [arXiv: 0905.4749] [INSPIRE].

[92] W. Beenakker et al., Soft-gluon resummation for squark and gluino hadroproduction, JHEP 12 (2009) 041 [arXiv:0909.4418] [INSPIRE].

[93] W. Beenakker et al., Supersymmetric top and bottom squark production at hadron colliders, JHEP 08 (2010) 098 [arXiv: 1006.4771] [INSPIRE].

[94] W. Beenakker et al., Squark and gluino hadroproduction, Int. J. Mod. Phys. A 26 (2011) 2637 [arXiv:1105.1110] [InSPIRE].

[95] ATLAS collaboration, Search for direct production of charginos, neutralinos and sleptons in final states with two leptons and missing transverse momentum in $p p$ collisions at $\sqrt{s}=8 \mathrm{TeV}$ with the ATLAS detector, JHEP 05 (2014) 071 [arXiv: 1403.5294] [INSPIRE].

[96] CMS collaboration, Search for top-squark pair production in the single-lepton final state in pp collisions at $\sqrt{s}=8 \mathrm{TeV}$, Eur. Phys. J. C 73 (2013) 2677 [arXiv:1308.1586] [INSPIRE].

[97] ATLAS collaboration, Search for direct production of charginos and neutralinos in events with three leptons and missing transverse momentum in $\sqrt{s}=8 \mathrm{TeV}$ pp collisions with the ATLAS detector, JHEP 04 (2014) 169 [arXiv: 1402.7029] [INSPIRE].

[98] D. Sengupta and S. Kulkarni, MadAnalysis 5 implementation of CMS-SUS-13-016, doi: 10.7484/INSPIREHEP.DATA.ZC3J.646F.

[99] CMS collaboration, Search for supersymmetry in pp collisions at $\sqrt{s}=8 \mathrm{TeV}$ in events with two opposite sign leptons, large number of jets, b-tagged jets, and large missing transverse energy, CMS-PAS-SUS-13-016 (2013).

[100] J. Alwall et al., The automated computation of tree-level and next-to-leading order differential cross sections and their matching to parton shower simulations, JHEP 07 (2014) 079 [arXiv:1405.0301] [InSPIRE]. 
[101] T. Sjöstrand, S. Mrenna and P.Z. Skands, PYTHIA 6.4 physics and manual, JHEP 05 (2006) 026 [hep-ph/0603175] [inSPIRE].

[102] DELPHES 3 collaboration, J. de Favereau et al., DELPHES 3, a modular framework for fast simulation of a generic collider experiment, JHEP 02 (2014) 057 [arXiv:1307.6346] [INSPIRE].

[103] M. Cacciari, G.P. Salam and G. Soyez, FastJet user manual, Eur. Phys. J. C 72 (2012) 1896 [arXiv:1111.6097] [InSPIRE].

[104] M. Cacciari, G.P. Salam and G. Soyez, The anti-kt jet clustering algorithm, JHEP 04 (2008) 063 [arXiv:0802.1189] [InSPIRE].

[105] Fermi-LAT collaboration, M. Ackermann et al., Searching for dark matter annihilation from Milky Way dwarf spheroidal galaxies with six years of Fermi Large Area Telescope data, Phys. Rev. Lett. 115 (2015) 231301 [arXiv:1503.02641] [InSPIRE].

[106] X.-J. Bi, X.-G. He and Q. Yuan, Parameters in a class of leptophilic models from PAMELA, ATIC and FERMI, Phys. Lett. B 678 (2009) 168 [arXiv:0903.0122] [INSPIRE].

[107] M. Cirelli and G. Giesen, Antiprotons from dark matter: current constraints and future sensitivities, JCAP 04 (2013) 015 [arXiv:1301.7079] [INSPIRE].

[108] ATLAS collaboration, Search for Higgs bosons decaying to aa in the $\mu \mu \tau \tau$ final state in $p p$ collisions at $\sqrt{s}=8 \mathrm{TeV}$ with the ATLAS experiment, Phys. Rev. D 92 (2015) 052002 [arXiv: 1505.01609] [INSPIRE].

[109] CMS collaboration, A search for pair production of new light bosons decaying into muons, Phys. Lett. B 752 (2016) 146 [arXiv:1506. 00424] [INSPIRE].

[110] N.-E. Bomark, S. Moretti, S. Munir and L. Roszkowski, A light NMSSM pseudoscalar Higgs boson at the LHC redux, JHEP 02 (2015) 044 [arXiv: 1409.8393] [INSPIRE].

[111] XENON1T collaboration, E. Aprile, The XENON1T dark matter search experiment, Springer Proc. Phys. 148 (2013) 93 [arXiv:1206.6288] [INSPIRE].

[112] F. Ruppin, J. Billard, E. Figueroa-Feliciano and L. Strigari, Complementarity of dark matter detectors in light of the neutrino background, Phys. Rev. D 90 (2014) 083510 [arXiv: 1408.3581] [INSPIRE].

[113] R.V. Harlander, S. Liebler and H. Mantler, SusHi: a program for the calculation of Higgs production in gluon fusion and bottom-quark annihilation in the standard model and the MSSM, Comput. Phys. Commun. 184 (2013) 1605 [arXiv:1212.3249] [INSPIRE].

[114] S. Liebler, Neutral Higgs production at proton colliders in the CP-conserving NMSSM, Eur. Phys. J. C 75 (2015) 210 [arXiv:1502.07972] [InSPIRE].

[115] CMS collaboration, Searches for electroweak production of charginos, neutralinos and sleptons decaying to leptons and $W, Z$ and Higgs bosons in pp collisions at $8 \mathrm{TeV}$, Eur. Phys. J. C 74 (2014) 3036 [arXiv: 1405.7570] [INSPIRE].

[116] CMS collaboration, Search for a pseudoscalar boson decaying into a $Z$ boson and the $125 \mathrm{GeV}$ Higgs boson in $\ell^{+} \ell^{-} b \bar{b}$ final states, Phys. Lett. B 748 (2015) 221 [arXiv: 1504.04710] [INSPIRE].

[117] ATLAS collaboration, Search for a CP-odd Higgs boson decaying to Zh in pp collisions at $\sqrt{s}=8 \mathrm{TeV}$ with the ATLAS detector, Phys. Lett. B 744 (2015) 163 [arXiv:1502.04478] [INSPIRE]. 
[118] CMS collaboration, Searches for heavy Higgs bosons in two-Higgs-doublet models and for $t \rightarrow$ ch decay using multilepton and diphoton final states in pp collisions at $8 \mathrm{TeV}$, Phys. Rev. D 90 (2014) 112013 [arXiv:1410.2751] [INSPIRE].

[119] LHC Higgs Cross Section Working Group collaboration, J.R. Andersen et al., Handbook of LHC Higgs cross sections: 3. Higgs properties, arXiv:1307.1347 [INSPIRE].

[120] V. Barger, T. Han and D.G.E. Walker, Top quark pairs at high invariant mass: a model-independent discriminator of new physics at the LHC, Phys. Rev. Lett. 100 (2008) 031801 [hep-ph/0612016] [INSPIRE].

[121] A. Djouadi, L. Maiani, A. Polosa, J. Quevillon and V. Riquer, Fully covering the MSSM Higgs sector at the LHC, JHEP 06 (2015) 168 [arXiv: 1502.05653] [INSPIRE].

[122] G. Bélanger, D. Ghosh, R. Godbole and S. Kulkarni, Light stop in the MSSM after LHC Run 1, JHEP 09 (2015) 214 [arXiv:1506.00665] [INSPIRE].

[123] ATLAS collaboration, Search for top squark pair production in final states with one isolated lepton, jets and missing transverse momentum in $\sqrt{s}=8 \mathrm{TeV}$ pp collisions with the ATLAS detector, JHEP 11 (2014) 118 [arXiv: 1407.0583] [INSPIRE].

[124] B. Dumont, B. Fuks and C. Wymant, MadAnalysis 5 implementation of CMS-SUS-13-011: search for stops in the single lepton final state at $8 \mathrm{TeV}$, doi: 10.7484/INSPIREHEP.DATA.LR5T.2RR3.

[125] ATLAS collaboration, Search for the direct production of charginos, neutralinos and staus in final states with at least two hadronically decaying taus and missing transverse momentum in pp collisions at $\sqrt{s}=8 \mathrm{TeV}$ with the ATLAS detector, JHEP 10 (2014) 096 [arXiv:1407.0350] [INSPIRE]. 